\title{
About the Use of Quality Indicators to Reduce Information Loss When Performing Upscaling
}

\author{
Christophe Preux \\ IFP Energies nouvelles, 1-4 avenue de Bois-Préau, 92852 Rueil-Malmaison Cedex - France \\ e-mail: christophe.preux@ifpen.fr
}

\begin{abstract}
Hydrocarbon reservoirs are characterized by the spatial distributions of petrophysical properties. These spatial characteristics are usually derived from well data and seismic information. To study a reservoir, the engineers build a fine geological model, also called a geostatistical model, to represent the field. The purpose is to capture as well as possible the peculiarities and heterogeneity of the true reservoir. At this stage, performing a flow simulation with such detailed geological models is just too time-demanding. Therefore, a possibility consists of upscaling the geological model to an upscaled mesh, thus resulting in a coarse reservoir model for which fluid flow can be numerically simulated in a reasonable amount of time. The coarse grid blocks of this reservoir model are attributed equivalent petrophysical properties related to the properties populating the fine grid blocks. These properties are upscaled, and so they do not capture all of the details of the fine model. In this paper, we investigate the potential of various numerical and easy to compute criteria, which help evaluate the information loss due to the upscaling process. Our final purpose is to provide and access the reliability of quality indicators, which make it possible to evaluate the quality of the upscaled reservoir model. The potential of this systematic and integrated study is illustrated with two types of numerical experiments based upon the SPE10 case. First, we apply different upscaling methods to determine coarse reservoir models. Quality indicators are computed for each of them so that we identify the most suitable upscaling methods. Then, the upscaled models are input to flow simulators to check the accuracy of our quality estimations. Second, we also investigate the influence of coarsening and try to determine from the computed quality indicators the coarse cell size above which too much information is lost.
\end{abstract}

\begin{abstract}
Résumé - À propos de l'utilisation d'indicateurs de qualité afin de réduire la perte d'information lors d'un upscaling - Les réservoirs pétroliers sont caractérisés par une distribution spatiale de propriétés pétrophysiques. Ces caractéristiques spatiales sont généralement dérivées de données de puits et sismiques. Les ingénieurs construisent alors un modèle géologique fin, aussi appelé modèle géostatistique, pour représenter le champ. Le but est de capturer aussi bien que possible les particularités et l'hétérogénéité du réservoir. À ce stade, réaliser une simulation d'écoulement avec ces modèles géologiques aussi détaillés est trop coûteux en temps de calcul. Par conséquent, une possibilité consiste à « upscaler » le modèle géologique sur une grille grossière, afin d'obtenir un modèle de réservoir grossier pour lequel l'écoulement peut être simulé numériquement dans un laps de temps raisonnable. On attribue alors des propriétés pétrophysiques équivalentes aux cellules de la grille grossière. Même si ces données équivalentes sont obtenues à partir des propriétés de la grille fine, elles ne tiennent pas compte de tous les détails du modèle géologique fin. Dans cet article, nous étudions le potentiel de
\end{abstract}


divers critères quantitatifs et facile à calculer, qui aident à évaluer la perte d'information due au processus d'upscaling. Notre but final est de fournir et d'évaluer des indicateurs de qualité, qui permettent d'évaluer la qualité du modèle de réservoir. L'intérêt de cette étude est illustrée avec deux types d'expériences numériques basées sur le cas SPE10. Tout d'abord, nous appliquons différentes méthodes d'upscaling afin d'obtenir différents modèles de réservoir. Les indicateurs de qualité sont calculés pour chacun d'eux afin d'identifier les méthodes d'upscaling les plus appropriées. Ensuite, les modèles réservoir sont fournis à un simulateur d'écoulement afin de vérifier l'exactitude de nos estimations de qualité. Deuxièmement, nous étudions également l'influence du nombre de mailles afin de déterminer à partir des indicateurs de qualité le nombre de maille limite à partir duquel trop d'information est perdue.

\section{INTRODUCTION}

\section{The Upscaling Process}

In the petroleum industry, the study of a field begins with the construction of a fine geological model. This model must capture the heterogeneity of the actual reservoir. As it is used to properly describe the spatial variations in petrophysical properties within the reservoir, reservoir engineers usually build high-resolution models with up to hundreds of millions of grid blocks. Inputting such models at once to flow simulators is not feasible in practice as it is extremely CPU time-demanding. Therefore, engineers are used to upscaling these geological models to upscaled meshes. The resulting coarse reservoir models represent the same field, but with fewer cells. The upscaling process consists in assigning equivalent petrophysical properties to the coarse grid blocks based upon the knowledge of the petrophysical properties populating the fine grid blocks. This has to be performed ensuring that fluid flow simulations for the fine or the coarse models yield very similar results. The usual properties to be upscaled are:

- discrete properties such as rock types or regions;

- continuous properties such as porosity, permeability or saturation.

In this paper, we focus on the upscaling of continuous properties, particularly permeability. The key assumption of the upscaling process is that fluid flow equations are the same on the fine (geological) and coarse (reservoir) scales. The upscaling process is split into two steps as shown in Figure 1:

- homogenization of the petrophysical properties;

- coarse discretization.

Clearly, the counterpart of upscaling is the information loss: upscaling tends to smooth fine heterogeneity features. This induces errors, which have to be quantified. Many upscaling methods exist to determine coarse reservoir models and many comprehensive reviews are available in the scientific literature [1-5]. In this paper, "upscaling" refers to an average in the physical space; the coarse permeability is also an equivalent (related to an average in the physical space) permeability and not an effective one (related to an average in the probability space). The notion of equivalent and effective permeability is discussed in [5].

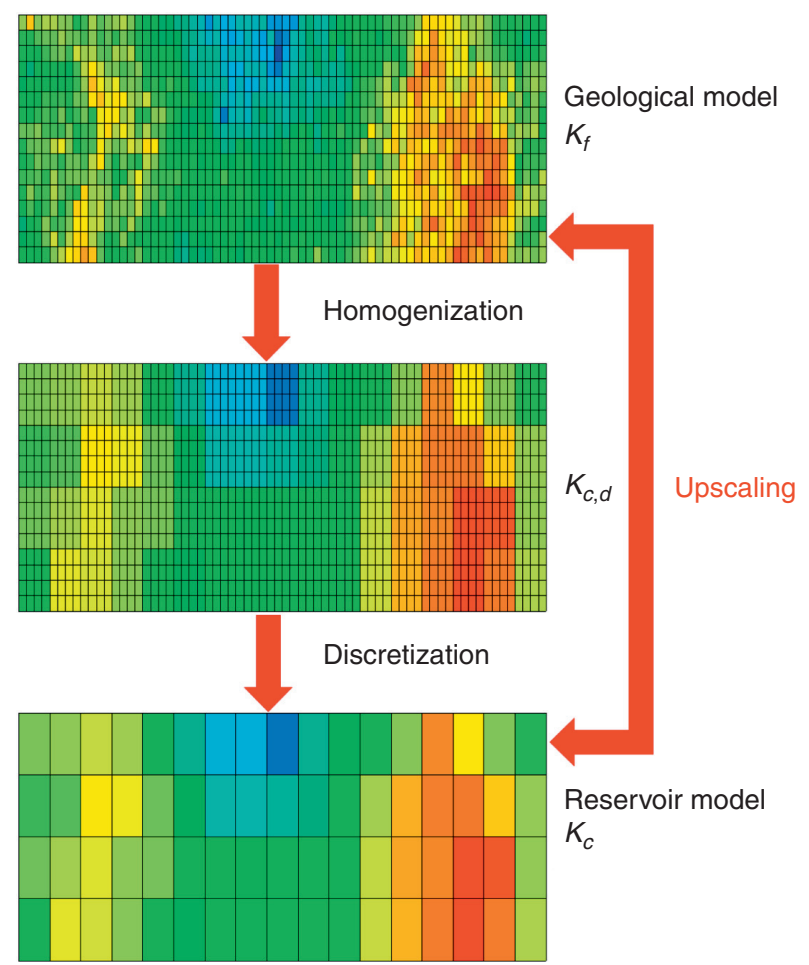

Figure 1

The two upscaling steps: the homogenization of the properties and the coarse discretization. 


\section{The Different Errors}

The two steps mentioned above, that is to say homogenization and discretization, imply different consequences [6]: homogenization smooths the permeability field and grid coarsening increases truncation errors. To study these different effects, Sablok and Aziz [6] define three kinds of errors:

- the total upscaling error;

- the discretization error due to grid coarsening;

- the smoothing error due to the smoothing of fine heterogeneity.

The total (or resulting) error is a non-intuitive combination of the two others: it is in no way a simple addition. This can be explained as follows. The discretization error and the smoothing error have different natures and can balance each other. This makes the analysis of information loss more complicated. In addition, error analysis is often based upon numerical fluid flow responses such as breakthrough time or production, which are complex functions of reservoir and simulation parameters: the multiphasic parameters such as relative permeability or capillary pressure are non-linear functions of saturation. Moreover, PVT parameters are non-linear functions of pressure. All these parameters make the error analysis very complex by fluid flow response. This is the reason why we look for quality criteria capable of handling these errors separately. Various authors [7-11] proposed different criteria to quantify these errors as recapped hereafter.

\section{OBJECTIVES OF THE PAPER}

In this paper, we first present different quality criteria. Then, we come back to the well-known SPE10 test case and introduce two numerical test cases. The first one aims to show that quality indicators can help identify the most suitable upscaling methods depending on the case studied. The second test case stresses the potential of these indicators to determine the level of coarsening above which too much information is lost. This level can be related to the representative elementary volume. Finally, we show how they can help reservoir engineers to select the most appropriate coarsening level.

\section{UPSCALING CRITERIA}

\subsection{The Upscaling Factor}

The upscaling factor [12, 13], also known as the upscaling ratio [14] or aggregation rate, is often used in reservoir simulation for upscaling purposes.
The dependence of upscaled permeability distribution as a function of the upscaling factor has been treated by several previous works $[15,16]$. It corresponds to the number of fine grid blocks, which are gathered all together to create a coarse grid block. It thus defines the coarsening level between the geological and the reservoir models. The computation of this factor is straightforward. With $N_{f}$ and $N_{c}$ the numbers of cells in the fine and coarse reservoir grids, respectively, the upscaling factor $(F u)$ is:

$$
F u=\frac{N_{c}}{N_{f}}
$$

The smaller the $F u$, the coarser the reservoir model. However, this factor usually depends on direction. Therefore, we denote $N_{f, x}, N_{f, y}, N_{f, z}$ and $N_{c, x}, N_{c, y}, N_{c, z}$ the numbers of cells in the geological and reservoir grids along directions $X, Y$ and $Z$. This leads to the following three upscaling factors as proposed by [8]:

$$
F u_{i}=\frac{N_{c, i}}{N_{f, i}} \quad \text { for } i=x, y, z
$$

As mentioned above, the upscaling factor following $[12,13]$ is the number of fine grid blocks in a coarse grid block. This definition is suitable provided the fine and coarse grids are Cartesian and the coarse grid is built by aggregating entire fine cells. However, as soon as the coarse grid is a Corner Point Geometry (CPG) grid, it no longer holds. In this case, the number of fine cells included in a coarse cell can vary depending on the target coarse cell. Thus, we prefer to refer to formulae 1 and 2 because they apply to any grids, whatever the coarse grid. This definition is very general and is independent of the upgridding method used to create the coarse grid $[17,18]$. Upgridding methods make it possible to adapt the gridding to geological heterogeneity. Therefore, the number of fine cells in a coarse reservoir cell is not constant per coarse cell. In addition, for a given upscaling factor, the impact of upscaling, in terms of information loss, will be less significant for a model with many fine cells than for a model with less fine cells. This motivated us to propose a new criterion, which allows us to aggregate more cells when there are many fine cells. This was already envisioned by [19], who investigated the impact of upscaling on a $3 \mathrm{D}$ model describing a field with meander belts and operated with steam-assisted gravity drainage.

\subsection{The Upscaling Extent Parameter}

It is quite intuitive that the higher the degree of coarsening, the greater the errors. To quantify the link between 
the upscaling factor and the information loss, [11] introduces the Upscaling Extent parameter, denoted $U E$. This was modified as follows by $[9,10]$ for practical purposes:

$$
U E=1-\frac{\ln \left(N_{c}\right)}{\ln \left(N_{f}\right)}
$$

Following this notation, when $U E=1$, the upscaled model is as coarse as possible and the resulting errors are potentially significant. On the other hand, when $U E=0$, the upscaled model is the same as the fine one and detailed information is fully preserved. We can propose an explicit relation between the upscaling factor and the upscaling extent parameter:

$$
\ln F u=-U E \ln \left(N_{f}\right)
$$

For a given upscaling method, the information loss is roughly proportional to the upscaling extent parameter [11]. Therefore, it is essential to evaluate the UE parameter when studying the potential of upscaling methods. Comparisons between different upscaling methods are meaningful provided the $U E$ levels are the same. Finally, the $U E$ concept can be propagated along axes $X, Y$ and $Z$, just like the upscaling factor [8]. In this case, we obtain:

$$
U E_{i}=1-\frac{\ln \left(N_{c, i}\right)}{\ln \left(N_{f, i}\right)} \quad \text { for } i=x, y, z
$$

\subsection{The Data Range Indicator}

Different studies stressed that upscaling removes extreme permeability values. It actually contributes to reducing large values and increasing small ones, which results in a narrower range, the range being the difference between the lowest and highest values. Based upon this analysis, [10] defined the ILIa Information Loss Indicator as:

$$
I L I a=\frac{R_{c}}{R_{f}}=\frac{\max _{c}(\mathrm{~K})-\min _{c}(\mathrm{~K})}{\max _{f}(\mathrm{~K})-\min _{f}(\mathrm{~K})}
$$

$R_{f}$ and $R_{c}$ are the ranges computed for the property of interest, that is permeability $K$, for the fine and the coarse models, respectively. Thus, according to this definition, no information is lost when $I L I a=1$, while all of it is lost when ILIa $=0$. Let us note that the upscaling extent parameter and the ILIa parameter vary inversely. In order to handle parameters all varying in the same way, we suggest using the ILIr Indicator ( $r$ for range):

$$
I L I r=1-\frac{R_{c}}{R_{f}}
$$

Thus, no information is lost when the ILIr parameter is 0 , whereas all of it is lost when it is 1 . This criterion makes it possible to control the range of values of the studied petrophysical property, but does not provide anything about their statistical properties. The following two sections investigate the potential of other criteria to address this issue.

\subsection{The variance Indicator}

[8] proposed another indicator, named the variance indicator, which is related to residuals. Briefly, residuals correspond to the difference between the coarse property values $K_{c, d}$ transferred to the fine grid and the fine property values $K_{f}$ (Fig. 1). This quantifies the error related to the smoothing of the heterogeneities observed on the fine scale. Thus, residuals are defined as:

$$
K_{r}=K_{f}-K_{c, d}
$$

The variance indicator corresponds to the variance of the residuals. In the case of a Cartesian grid, it is defined as:

$$
\sigma^{2}=E\left[(K-E(K))^{2}\right] \approx \frac{1}{N} \sum_{j=1}^{N}\left(K_{j}-\bar{K}\right)^{2}
$$

$\sigma^{2}$ and $E(x)$ are the variance and the mean of $K$, respectively. The sample mean or arithmetic mean of $K$ is denoted $\bar{K}$ and $N$ is the number of sampled values. The variance measures the dispersion of a distribution. The purpose of an indicator relative to variance is to check whether the variance of the property on the coarse scale is the same as its variance on the fine scale. Keeping this goal in mind, we focus on the variance of the residuals, which must be close to zero. We introduce the ILIV variance indicator:

$$
I L I v=\frac{E\left[\left(K_{r}-E\left(K_{r}\right)\right)^{2}\right]}{E\left[\left(K_{f}-E\left(K_{f}\right)\right)^{2}\right]}
$$


Thus, ILIV $=0$ when the variance of the residuals is zero (white noise). In this case, there is no information loss. Expanding residual variance yields:

$$
\begin{gathered}
E\left[\left(K_{r}-E\left(K_{r}\right)\right)^{2}\right] \approx \frac{1}{N} \sum_{j=1}^{N}\left(K_{r, j}-\bar{K}_{r}\right)^{2} \\
=\frac{1}{N} \sum_{j=1}^{N}\left(K_{f, j}-\bar{K}_{f}\right)^{2} \\
+\frac{1}{N} \sum_{j=1}^{N}\left(K_{c, d, j}-\bar{K}_{c, d}\right) \\
\left(\left(K_{c, d, j}-\bar{K}_{c, d}\right)-2\left(K_{f, j}-\bar{K}_{f}\right)\right)
\end{gathered}
$$

Clearly, the upscaling process tends to narrow the range of values. Thus:

$$
K_{c, d, j}-E\left(K_{c, d}\right) \leq 2\left(K_{f, j}-\bar{K}_{f}\right)
$$

As a result, the second term on the left-hand side of the equation is negative, meaning that:

$$
E\left[\left(K_{r}-E\left(K_{r}\right)\right)^{2}\right] \leq E\left[\left(K_{f}-E\left(K_{f}\right)\right)^{2}\right]
$$

This means that the ILIV indicator is less than 1 in most of the cases studied. When this indicator tends to 1 , the variance of residuals and the variance of the fine distribution are almost the same. This means that the upscaling process is too strong and that too much information is lost.

\subsection{The Cardwell and Parsons Bounds}

This section comes back to the bounds proposed by Cardwell and Parsons [20]. These bounds were derived from a variational principle [21]. They are written as (Fig. 2):

$$
\begin{aligned}
& K_{c, x}^{\min }=\mu_{a}^{z}\left(\mu_{a}^{y}\left(\mu_{h}^{x}\left(K_{f, x}\right)\right)\right) \\
& K_{c, x}^{\max }=\mu_{h}^{x}\left(\mu_{a}^{z}\left(\mu_{a}^{y}\left(K_{f, x}\right)\right)\right)
\end{aligned}
$$

where:

- $\mu_{a}^{i}$ is the arithmetic mean along direction i;

- $\mu_{h}^{i}$ is the harmonic mean along direction $i$.

The interested reader can refer to [20] and [22] for detailed formulations in the case of Cartesian grids. Let us consider CPG grids and denote $v_{i, j, k}$ the volume

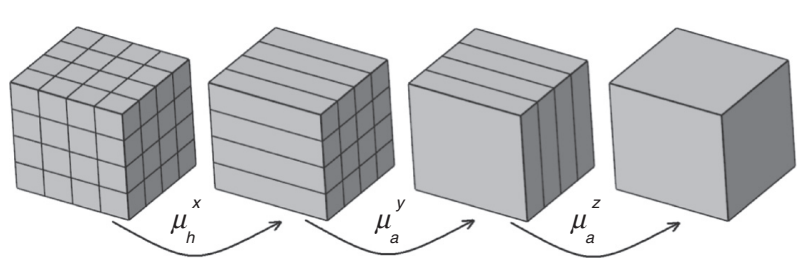

Figure 2

Lower bound computation.

of a fine cell. We assume that a coarse cell consists of $N_{x} N_{y} N_{z}$ cells and define:

$$
\begin{gathered}
V_{i, j}=\sum_{i=1}^{N_{x}} v_{i, j, k} \\
V_{k}=\sum_{j=1}^{N_{y}} V_{j, k}
\end{gathered}
$$

$$
V=\sum_{k=1} N_{z} V_{k}
$$

This leads to the following expression for $K_{c, x}^{\mathrm{min}}$ :

$$
K_{c, x}^{\min }=\frac{1}{V} \sum_{k=1}^{N_{z}} \sum_{j=1}^{N_{y}} V_{j, k} \frac{V_{i, k}}{\sum_{i=1}^{N_{x}} \frac{v_{i, j, k}}{k_{i, j, k}}}
$$

Therefore, referring to Cardwell and Parsons bounds, we define the Cardwell and Parsons indicator:

$$
\text { Error }_{i}=\log _{10} \frac{K_{c, i}^{\max }}{K_{c, i}^{\min }}
$$

This provides a grid property, that can be used to anticipate where the approximation of the upscaled permeability is poor. When Error $=0, K_{c, i}^{\min }=K_{c, i}^{\max }$ and the upscaling is trivial. When it is $1, K_{c, i}^{\max }$ is ten times larger than $K_{c, i}^{\min }$.

\subsection{The QQplot}

Sablok and Aziz [6] stressed the usefulness of QQplots [7] to analyze information loss. This is especially true when the stochastic realizations used to represent petrophysical 
property variations are not derived from two-point statistics methods. QQplots make it possible to compare at once the distribution on the coarse scale with the distribution on the fine scale. To build the QQplot between $K_{f}$ and $K_{c, d}$, we proceed as follows. First, we sort all $K_{f}$ and $K_{c, d}$ values in ascending order. These distributions having the same number of values, we note $K_{f}^{\text {sort }}$ and $K_{c, d}^{\text {sort }}$ the sorted distributions of $K_{f}$ and $K_{c, d}$. The QQplot is the discrete function:

$$
K_{f}^{s o r t} \rightarrow K_{c, d}^{s o r t}
$$

If we compare two identical distributions, we obtain the bisector. Thus, the more accurate the upscaling process, the closer the QQplot to the bisector.

\section{UPSCALING METHODS}

In this section, we recap the basics of the upscaling methods applied in this paper. The interested reader can refer to [3] for a detailed review.

\subsection{Basic Algebraic Methods}

We note $V$ and $v$ the volume of the coarse and fine cells, $K$ and $k$ the permeability of the coarse and fine cells and $J$ the set of fine cells contained in the coarse cell:

In such conditions, the arithmetic mean is written as:

$$
K_{i}^{\text {arith }}=\frac{1}{V_{i}} \sum_{j \in J} v_{j} k_{j}
$$

While the harmonic mean is:

$$
\frac{1}{K_{i}^{\text {harmo }}}=\frac{1}{V_{i}} \sum_{j \in J} \frac{v_{j}}{k_{j}}
$$

Another useful simple algebraic average is the geometric mean:

$$
K_{i}^{\text {geom }}=\frac{1}{V_{i}} \prod_{j \in J} k_{j}^{\frac{v_{j}}{V_{j}}}
$$

\subsection{More Sophisticated Algebraic Methods}

The following three upscaling methods are more complex:

\section{The Power-Law Method}

Journel et al. [23] introduced the power-law method, which can be written as:

$$
K_{c}=\frac{1}{V_{c}}\left(\int_{V_{c}} K(x)^{p} d V\right)^{\frac{1}{p}}
$$

with $p \in[-1,1]$. When $p$ is $-1,0$ and 1 , the power-law method is equivalent to the harmonic mean, the geometric mean and the arithmetic mean, respectively. A study of this method and a determination of the power exponent are available in [24].

\section{The Algebraic Isotropic Method}

The algebraic isotropic method [25] is rooted in the Cardwell and Parsons approach [20]. In [25], Guérillot et al. proposed computing the geometric mean of the two Cardwell and Parsons bounds:

$$
K_{x}^{\text {Alg.Iso. }}=\sqrt{K_{c, i}^{\min } K_{c, i}^{\max }}
$$

$K_{c, x}^{\min }$ and $K_{c, x}^{\max }$ are given by Equations (13) and (14).

\section{The Bound Combination Method}

Duquerroix et al. [26] and Kruel-Romeu [27] suggested to generalizing the algebraic isotropic method to account for field anisotropy. The upscaled properties are then derived from the following relationship, known as the bound combination method:

$$
K_{x}^{B C}=\sqrt[6]{\left(K_{c, x}^{\min }\right)^{2}\left(K_{c, x}^{\max }\right)^{2} K_{c, x}^{3} K_{c, x}^{4}}
$$

The two additional terms, compared with Equation (25), are estimated from the following expressions:

$$
K_{c, x}^{3}=\mu_{a}^{y}\left(\mu_{h}^{x}\left(\mu_{a}^{z}\left(K_{f, x}\right)\right)\right)
$$

$$
K_{c, x}^{4}=\mu_{a}^{z}\left(\mu_{h}^{x}\left(\mu_{a}^{y}\left(K_{f, x}\right)\right)\right)
$$

Notation $\mu$ stands for mean. The $a$ and $h$ subscripts specify whether this mean is arithmetic or harmonic.

\section{The Numerical Local Method}

The last upscaling method considered hereafter is the numerical local method. This one is based upon the simulation of Darcy flow with given boundary conditions for each group of fine grid blocks within coarse grid blocks. In this paper, we use no-flow boundary conditions. Flow is assumed to be linear and steady-state. This method is described in several papers [28-31]. It was shown to provide satisfactory results provided the coarse cell size is very large compared with the correlation length of permeability [32]. 


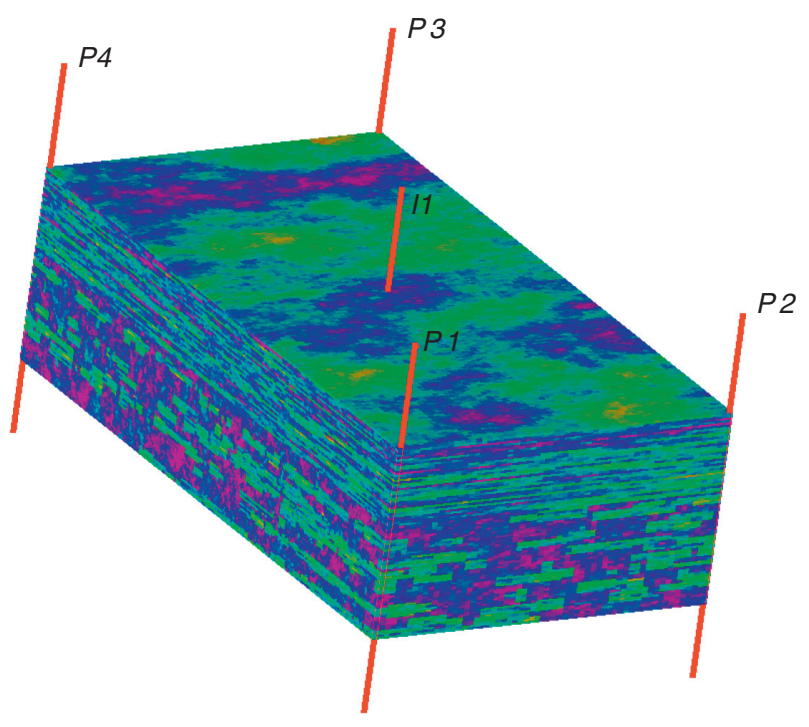

Figure 3

Porosity distribution within the whole SPE10 model.

\section{SENSITIVITY STUDY AND NUMERICAL EXPERIMENT}

\subsection{The SPE10 Test Case [33]}

In this section, we consider the second case of the Tenth SPE Comparative Solution [12]. The geological model consists of part of a Brent sequence. It includes two units. The top part of the model (21.336 m (70 ft), 35 layers) belongs to the Tarbert formation and corresponds to a prograding near-shore environment. Its lower part (Upper Ness, $30.48 \mathrm{~m}$ (100 ft), 50 layers) is fluvial. The geometry of this model is very simple: there is no top structure or fault. The fine scale model is discretized over a regular Cartesian grid with $60 \times 220 \times 85$ grid blocks (i.e. $1.122 \times 10^{6}$ grid blocks). The total dimensions are $365.76 \times 670.56 \times 51.816 \mathrm{~m}^{3} \quad\left(1200 \times 2200 \times 170 \mathrm{ft}^{3}\right)$ with grid blocks of $6.096 \times 3.048 \times 0.6096 \mathrm{~m}^{3}$ $\left(20 \times 10 \times 2 \mathrm{ft}^{3}\right)$. The production scheme is based upon a classical inverted " 5 -spot" pattern with four producers $P$ located in the corners and an injector $I$ in the center (Fig. 3). The coordinates of the wells are ( $i$ : $X$-coordinate, $j: Y$-coordinate):

- $P_{1}: i=0, j=0$;

- $P_{2}: i=60, j=0$;

- $P_{3}: i=60, j=220$;

- $P_{4}: i=0, j=220$

$-I: i=30, j=110$.

All wells are vertical and completed throughout the whole formation (Fig. 3). The productivity index of each
TABLE 1

Oil PVT table

\begin{tabular}{c|c|c}
\hline Pressure (bar) & $B_{\mathrm{o}}$ & $\mu_{\mathrm{o}}$ \\
\hline 20.68 & 1.05 & 2.85 \\
\hline 55.13 & 1.02 & 2.99 \\
\hline 551.34 & 1.01 & 3.0 \\
\hline
\end{tabular}

well is computed using the Peaceman method. The injection rate is $795 \mathrm{~m}^{3} /$ day (5 $000 \mathrm{bbl} /$ day) in reservoir conditions with a maximum injection bottom-hole pressure of $689.18 \mathrm{bar}(10000 \mathrm{psi})$. The four producers produce at 275.67 bar (4 000 psi) bottom-hole pressure. All wells have an internal diameter of $0.3048 \mathrm{~m}(1.0 \mathrm{ft})$.

The water properties are:

- formation volume factor $B_{w}=1.01$;

- compressibility $c_{w}=4.5 \times 10^{-5} \mathrm{bar}^{-1}$;

- viscosity $\mu_{w}=0.3 \mathrm{cp}$.

On the other hand, we consider a dead oil with the PVT properties reported in Table 1.

The relative permeabilities are derived from the Brooks-Corey models [34]:

$$
\begin{aligned}
& k_{r w}=\left(\frac{S-S_{w i}}{1-S_{w i}-S_{o r}}\right)^{2} \\
& k_{r 0}=\left(\frac{1-S-S_{w i}}{1-S_{w i}-S_{o r}}\right)^{2}
\end{aligned}
$$

where $S_{w i}=S_{\text {or }}=0.2$.

The upscaling and flow simulation results presented hereafter were obtained using the CobraFlow software and the PumaFlow simulator [35, 36], respectively. In this work, we did not study the numerical dispersion due to the mathematical model of the simulator. Upscaling and coarsening cause numerical dispersion. In this work, the simulator adapts the time step itself to obtain correct results. To study the numerical dispersion precisely, we should study the mathematical method used in the simulator and using a constant CFL criterion [37].

\subsection{How Important is the Choice Of Upscaling Method?}

The spatial distribution of petrophysical properties within the two SPE10 units is very different (Fig. 3). On the other hand, it is essential to consider a stationary distribution for investigating the influence of upscaling methods on information loss. We first attempted to apply the various criteria defined above without decoupling the two units, which made the interpretation and 
TABLE 2

Upscaling factor [8]

\begin{tabular}{c|c|c|c}
\hline Global & $X$ & $Y$ & $Z$ \\
\hline 0.0125 & 0.25 & 0.25 & 0.2 \\
\hline
\end{tabular}

TABLE 3

Upscaling extent parameter [10]

\begin{tabular}{c|c|c|c}
\hline Global & $X$ & $Y$ & $Z$ \\
\hline 0.3360 & 0.3386 & 0.2570 & 0.4527 \\
\hline
\end{tabular}

understanding of the results very difficult. A few tests pointed out that it is required to estimate the upscaling criteria on each unit separately when there are significant differences in petrophysical properties. As a result, we focus on the top part of the SPE10 model. The following subsections are dedicated to the comparison of different upscaling methods referring to the criteria defined above. This analysis makes it possible to select the best method to use. In all cases studied, the coarse scale reservoir grid is fixed.

\subsubsection{Upscaling Methods Used}

The reservoir grid encompasses $15 \times 55 \times 17$ cells. We consider the 7 upscaling methods introduced in Section 2:

- arithmetic mean;

- harmonic mean;

- geometric mean;

- power-law method;

- algebraic isotropic method;

- bound combination method;

- numerical local method.

The upscaling factor and upscaling extent parameter computed for the case studied are reported in Tables 2 and 3 . These parameters have the same values whatever the upscaling method.

The upscaling extent parameter (Tab. 3) is higher along direction $Z$. This suggests that upscaling along this direction is likely to be more problematic. The analysis of the upscaling factor leads to a similar conclusion. Its value is smaller along direction $Z$ (Tab. 2), meaning that coarsening is stronger in this direction.

\subsubsection{The Cardwell and Parsons Bounds}

The results derived from the Cardwell and Parsons bounds (Eq. 19) are recapped hereafter. Figure 4 shows that the $X$-and $Y$-permeability errors are very similar as
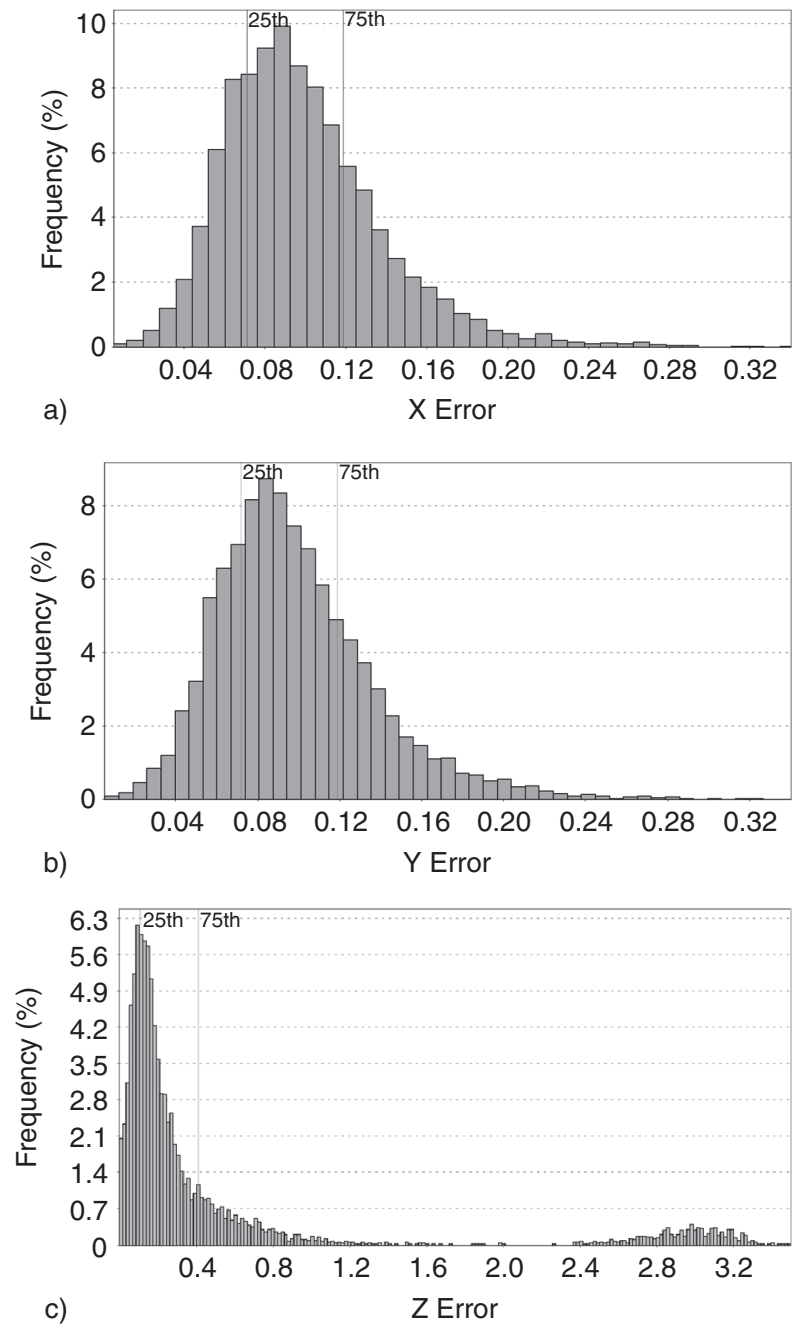

Figure 4

Histograms of the Cardwell and Parsons errors computed for $a, b)$ the horizontal permeabilities and c) the vertical permeabilities.

$K_{x}^{f}=K_{y}^{f}$ for the SPE10 test case. In addition, the upscaling factors are the same for both $X$ and $Y$ axes (Tab. 2). The slight differences evidenced from Figure 4 result from the dimensions of the coarse cells which vary with direction. Generally speaking, the error is small. In terms of percentiles, $75 \%$ of the grid blocks show Cardwell and Parsons errors of less than 0.12 for horizontal permeabilities. This means that the coarsening effect induced by upscaling is not too strong, or in other words, that heterogeneity is moderate in every grid block. The results are clearly different for vertical permeabilities $\mathrm{Kz} .75 \%$ of the cells show an error of less than 0.41 and $25 \%$ of them less than 0.11 . Basically, $25 \%$ of the grid blocks are characterized by an error greater than 0.41 . 


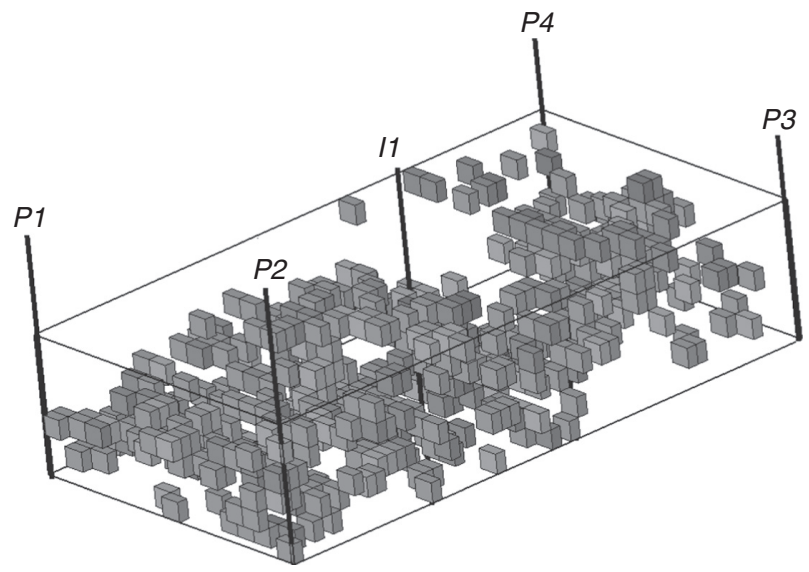

Figure 5

Localization of the grid blocks with Cardwell and Parsons errors for vertical permeabilities larger than 2 .

This means that the upper Cardwell and Parsons bound is 2.57 times larger than the lower bound for $25 \%$ of cells. In addition, a second peak is evidenced with errors in the range between 2.4 and 3.3: all these cells correspond to an upper bound 251 times larger than the lower bound. These values are very high and stress that the 0.2 upscaling factor applied along the $Z$ axis is too significant as it is (without any specifically designed upgridding technique). Moreover, in the $Z$ direction, the permeability is more heterogeneous: the permeability is organized on fine layers with very different permeability. Therefore, any upscaling technique can be applied when dealing with the horizontal directions for the case studied. However, the vertical upscaling has to be performed more carefully. Figure 5 shows the locations of the grid blocks with $Z$ error values higher than 2 . At first glance, we observe that they can be anywhere, which once again highlights the difficulty in determining an appropriate technique for upscaling the vertical permeabilities.

\subsubsection{Variance, Data Range Indicators and QQplots}

The data range and variance indicators, denoted ILIr and ILIV as explained in the previous sections, are displayed in Figure 6. A general comment is that both ILIr and ILIV indicators evolve the same way whatever the upscaling technique applied.

Whatever the direction of interest, the data range and variance indicators are minimal for the arithmetic method and maximal for the harmonic method. This feature is also evidenced for the QQplots, especially for vertical permeabilities. All errors are bounded by those

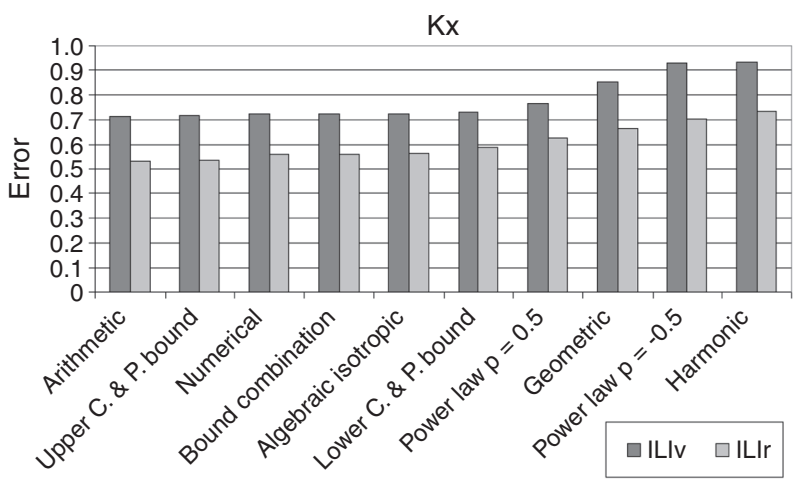

a)

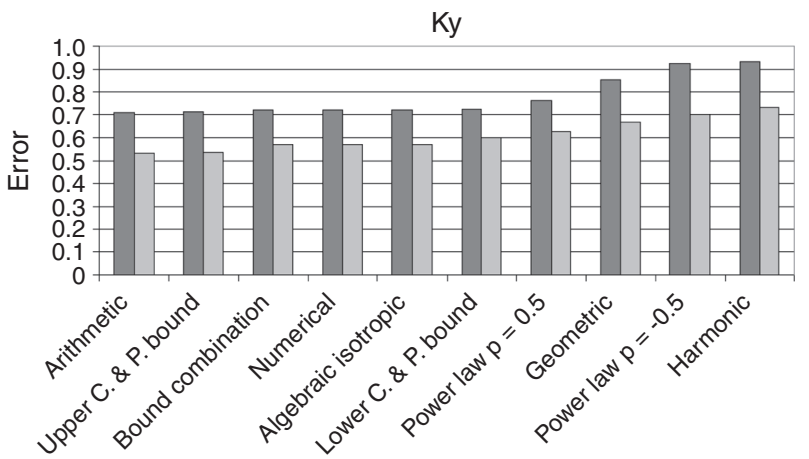

b)

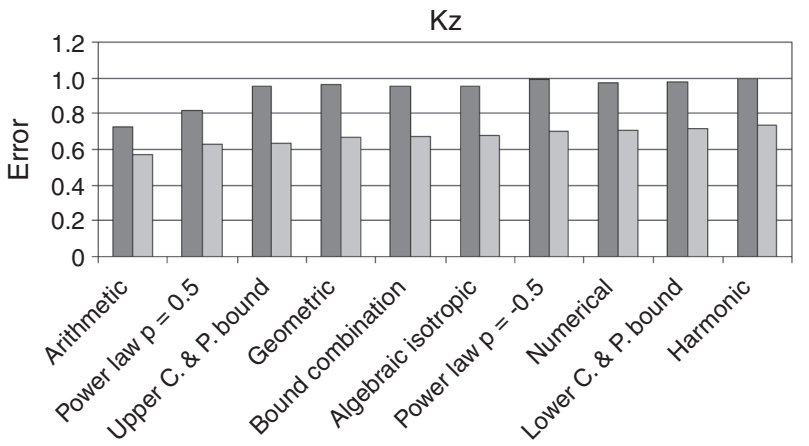

c)

Figure 6

Data range and variance indicators, respectively ILIr and ILIV, for $\mathrm{a}, \mathrm{b})$ the horizontal permeabilities and c) the vertical permeabilities derived from different upscaling methods.

estimated for the arithmetic and harmonic methods. The indicators also respect these bounds, also called fundamental inequality or Wiener bounds [38], because they are always valid $[3,20,39,40]$. Thus, in the case studied, the data range and variance indicators can be considered as simply-computed criteria, which allow us to rank the various upscaling methods. Another point is that the bound combination and the algebraic methods yield similar results. For the example studied, the horizontal 

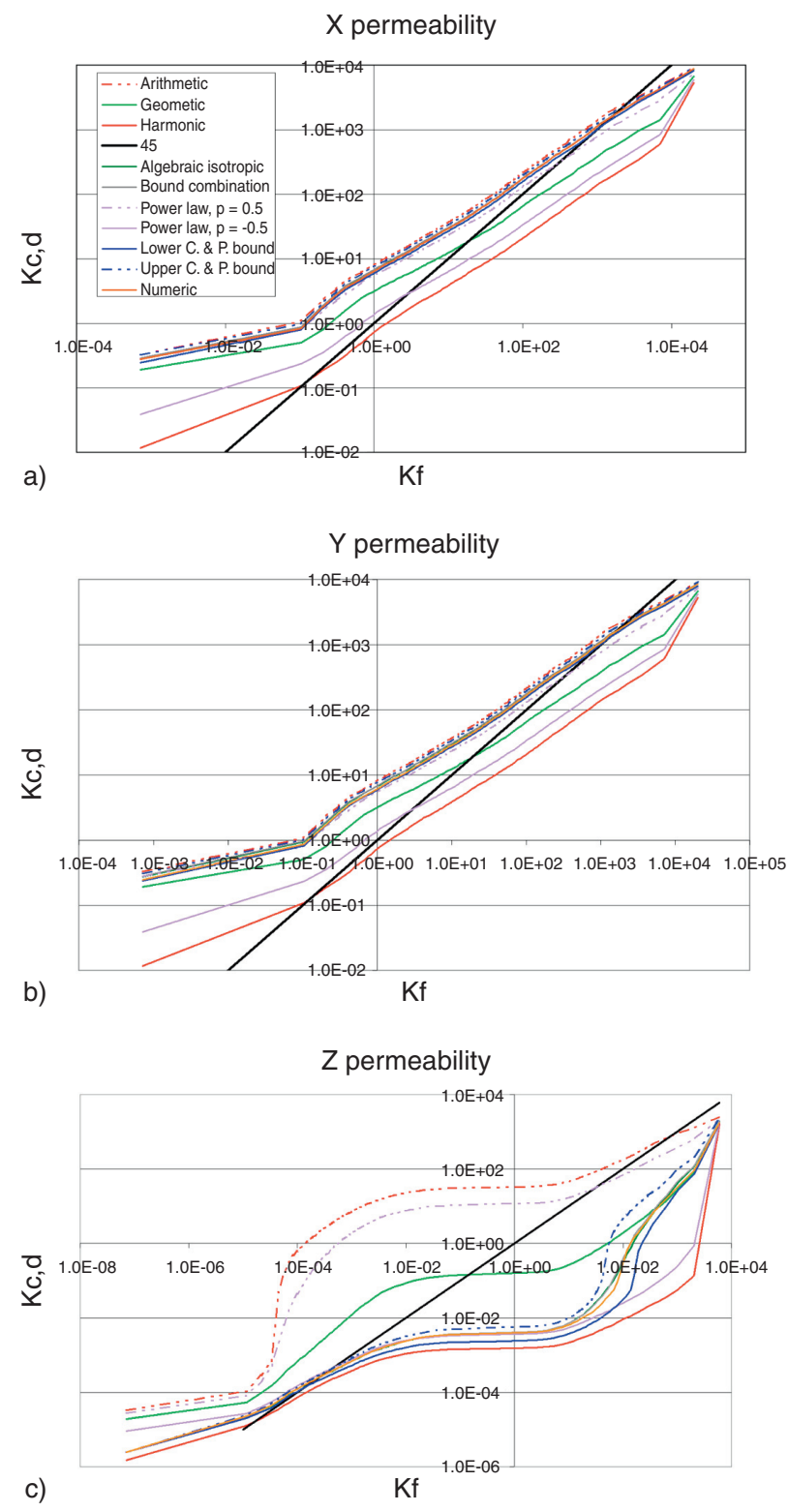

Figure 7

QQplots for $\mathrm{a}, \mathrm{b}$ ) the horizontal permeabilities and c) the vertical permeabilities derived from different upscaling methods.

permeability is isotropic: $K_{x}$ is the same as $K_{y}$. In this special case, the two methods are similar. Now, in Figure 7, we can analyze the results given by the Cardwell and Parsons bounds on the QQplot. These bounds define an envelope, narrower than the one derived from the harmonic and arithmetic methods. According to Cardwell and Parsons [20] and Le Loch [41], an accurate estimate of the upscaled permeability should range between the
Cardwell and Parsons bounds. This also holds for the data range and the variance indicators as well as the QQplots. Also, as explained above, a good method is expected to be associated with zero data range and variance indicators. Moreover, if we study the result given by the power-law method for different values of $p[p=-1$ (harmonic), $p=-0.5, p=0.5, p=1$ (arithmetic)], we note that the results are included in the envelope given by the Wiener bounds. So, an appropriate method consists of fitting the exponent $p$ to reduce the difference between the QQplot curve and the bisector. The analysis of these various criteria must help us select an appropriate upscaling method. In addition, it is worth noting that the numerical local upscaling method yields for $K_{x}$ and $K_{y}$ the same results as the bound combination and the algebraic isotropic methods. Such behavior was actually expected. The two latter techniques are known to be very close to the numerical local method. However, if considering especially $K_{z}$ (Fig. 6), it is shown that the numerical local method departs from the results derived from the bound combination and the algebraic isotropic methods. Considering the QQplots on top of the data range, variance indicators can contribute to refining our choice of the upscaling method. In Figure 7, we observe that the harmonic average tends to reduce the upscaled values, while the arithmetic method leads to the opposite behavior. In this case, the differences between the numerical local method and the bound combination and algebraic isotropic methods are very small. The three curves turn out to be almost the same for both $K_{x}$ and $K_{y}$. On the other hand, the numerical local method lightly departs from the two algebraic methods for $K_{z}$. In Sections 3.2.1 and 3.2.2, we checked that the upscaling of vertical permeabilities is more challenging than the upscaling of horizontal permeabilities. The analysis of the indicators that we suggested here lead us to the same conclusion. Whatever the upscaling method, the data range and variance indicators are larger for vertical permeabilities than for horizontal permeabilities. In addition, the area between the QQplots for the two Cardwell and Parsons bounds is larger for vertical permeabilities than for horizontal permeabilities. To consolidate these conclusions, we decided to move one step ahead and confront the simulation results.

\subsubsection{Convergence of the Quality Indicators and the Simulation Results}

Figure 8 depicts the bottom-hole pressure at the injector. For three upscaling methods (geometric, harmonic and power-law with an exponent of -0.5 ), the injector reaches the maximal bottom-hole pressure. The harmonic method provides a lower bound for permeability, 


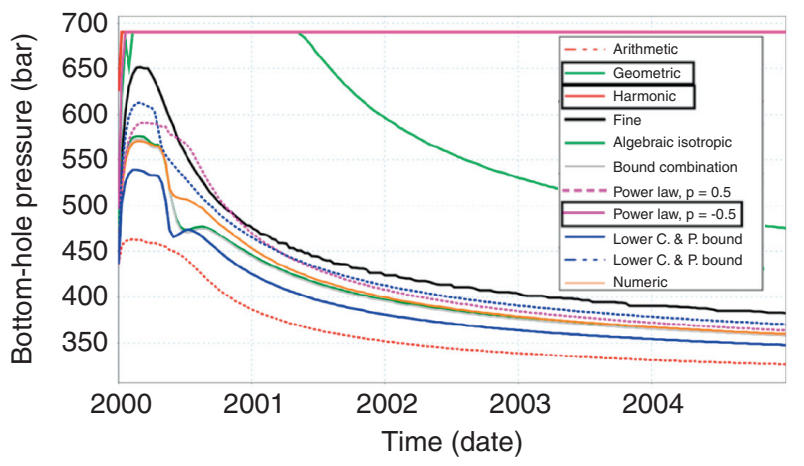

Figure 8

Comparison of the bottom-hole pressures at the injector for reservoir models derived from different upscaling methods (black $=$ fine grid results).

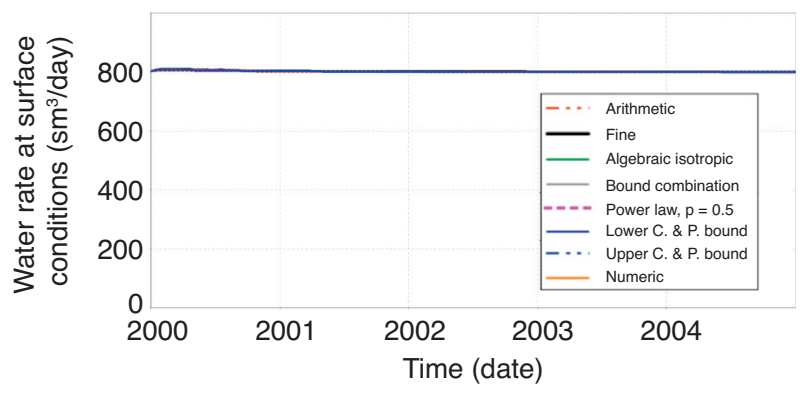

Figure 9

Comparison of the water injection rates at surface conditions for reservoir models derived from different upscaling methods (black $=$ fine grid results).

the upscaled productivity index for the injector is also too low and the pressure drops. The same conclusion can be established for the power-law method with an exponent of -0.5 . Coming back to the QQplots in Figure 7, we note that these methods should not be recommended in this case: these three methods give very different QQplots compared with the others (for $K x$ and $K y$ ). In such conditions, there is no way to make valuable comparisons with these three methods.

The water injection rates at surface conditions obtained for different upscaling methods are plotted in Figure 9. Excluding these three methods, we have a constant injection rate. We can also make valuable comparisons with these seven other methods. In conclusion, we can note that if the upscaling method is not chosen intelligently, the upscaling process can drastically change the well behavior and mislead the reservoir engineer. The water-oil ratios simulated for different reservoir models obtained from different upscaling methods are plotted in Figure 10.

The increase in the water-oil ratio by the arithmetic method is not always noticeable (see, for instance, wells P1, P3 and P4). It is known that for isotropic fields, the bound combination method and the algebraic isotropic method give the same results for both indicators and QQplots. Now, focusing on the simulated water-oil ratios, an anomalous behavior is evidenced for wells P3 and P4. The results at well P3 obtained for the upscaled models are all wrong compared with the fine model. The overall behavior of the ratio is not respected. On the other hand, the curves obtained for well P4 evolve the same way, but the water-oil ratios are underestimated. This may be related to the too large value of the upscaling factor. In this special case, it seems that the most relevant methods are the bound combination and the algebraic methods, particularly when considering wells P1 and P4. However, none of these methods makes it possible to perfectly reproduce the results simulated for the geological model. A surprising result is pointed out for the numerical local method. Clearly, this method does not yield the best approximation. For wells P3 and P4, the results obtained are very close to those derived from the bound combination and the algebraic methods. However, for the other two wells, they are very different. The water-oil ratio is overestimated for well $\mathrm{P} 1$, while it is the opposite for well P4. This stresses the difficulties in selecting a suitable upscaling method.

The results in terms of oil rates are reported in Figure 11. In Figure 7, we observed that the powerlaw method with an exponent of 0.5 gives results very similar to those derived from the arithmetic average. The permeabilities derived from these two methods (specially $K_{z}$ ) are slightly higher compared with the bisector. The same phenomenon is shown for oil rates. Overall, for all wells, the methods identified as best in the previous sections (bound combination, algebraic and numerical local) overestimate oil rates. More precisely, the two algebraic methods give similar results. The numerical local method gives a correct result for oil rates, that is a result very close to those provided by the two algebraic methods. If referring to well P1, the arithmetic average and the power-law method with an exponent of 0.5 drastically overestimate the oil rates. Coming back to the QQplots in Figure 7, we note that these methods should not be recommended in this case. Therefore, the QQplot results are in good agreement with the flow results simulated for the coarse reservoir models. In conclusion, it seems that the bound combination, algebraic isotropic and numerical methods are the best choice. 


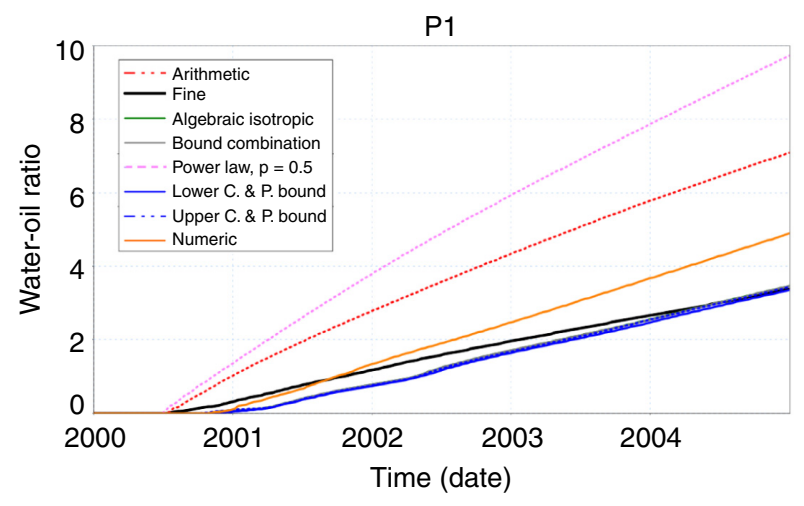

P2

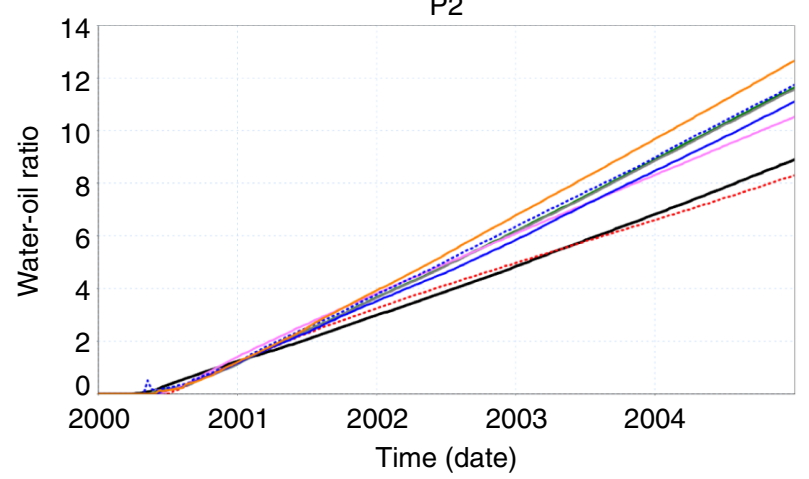

P3

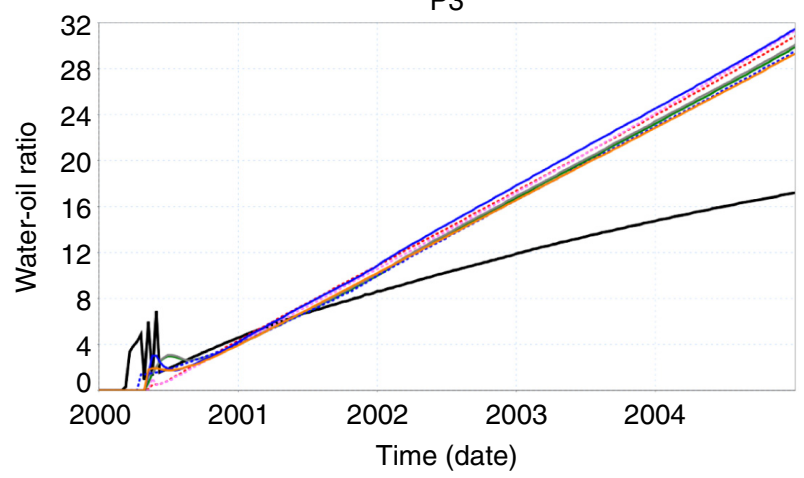

P4

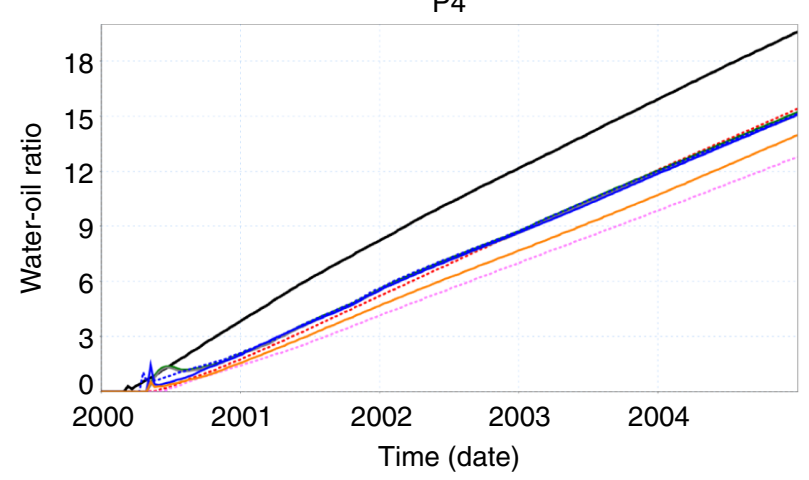

Figure 10

Comparison of the water-oil ratios for reservoir models derived from different upscaling methods (black $=$ fine grid results).
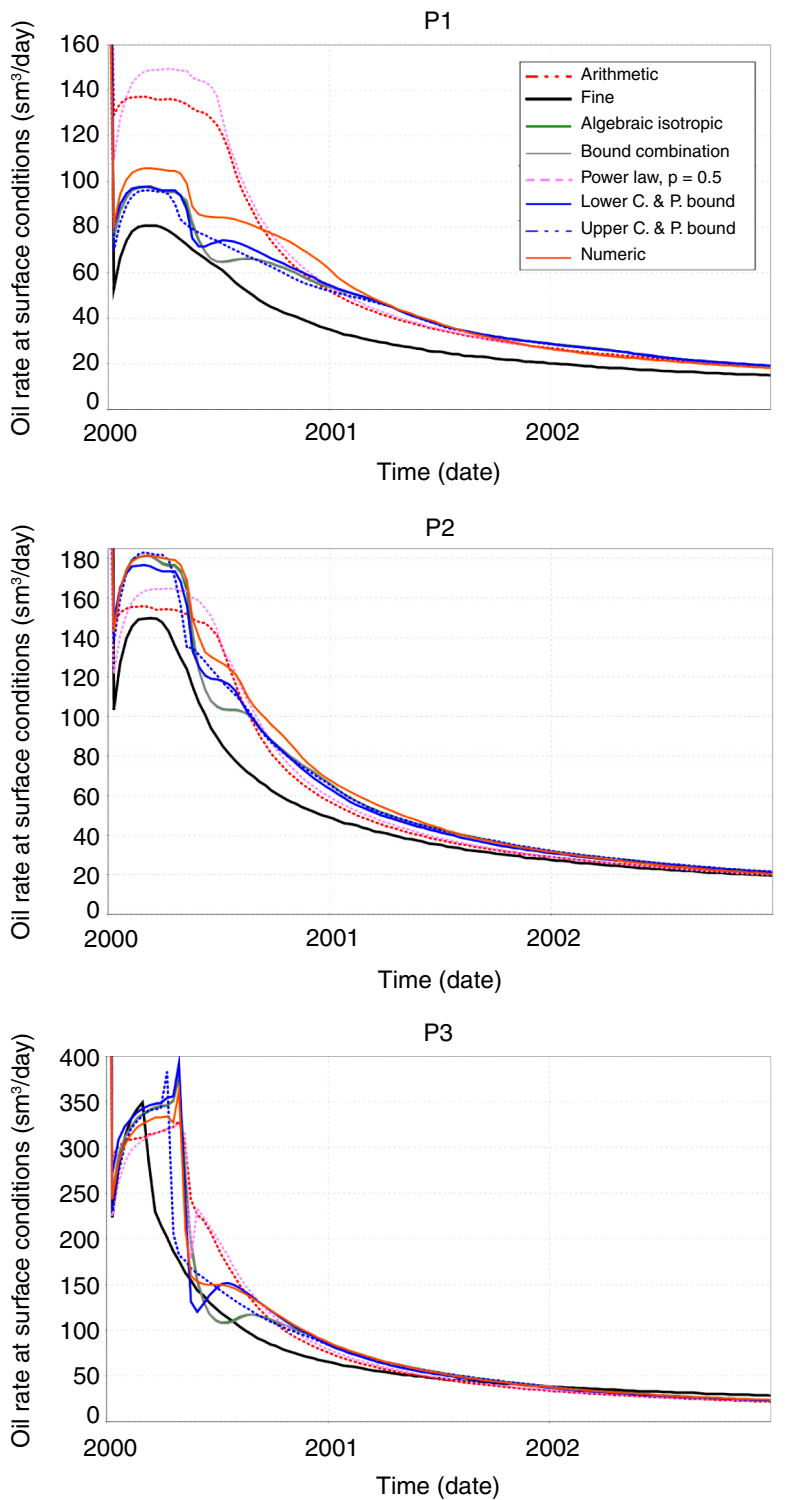

P4

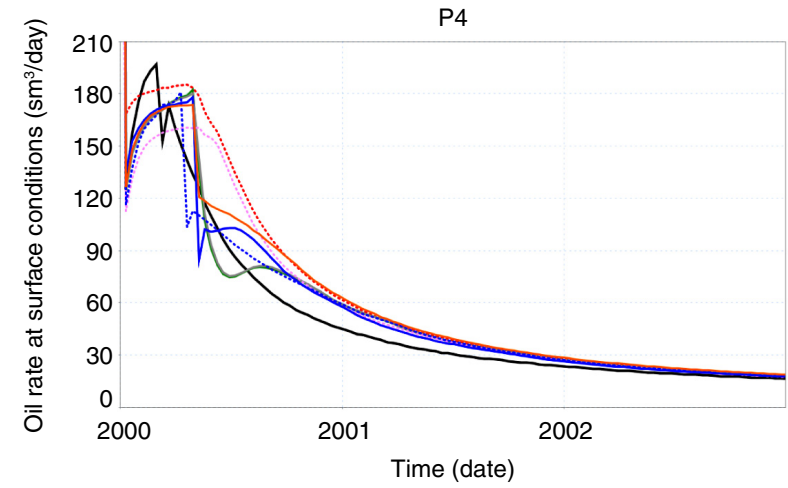

Figure 11

Comparison of the oil rates at surface conditions for reservoir models derived from different upscaling methods. 

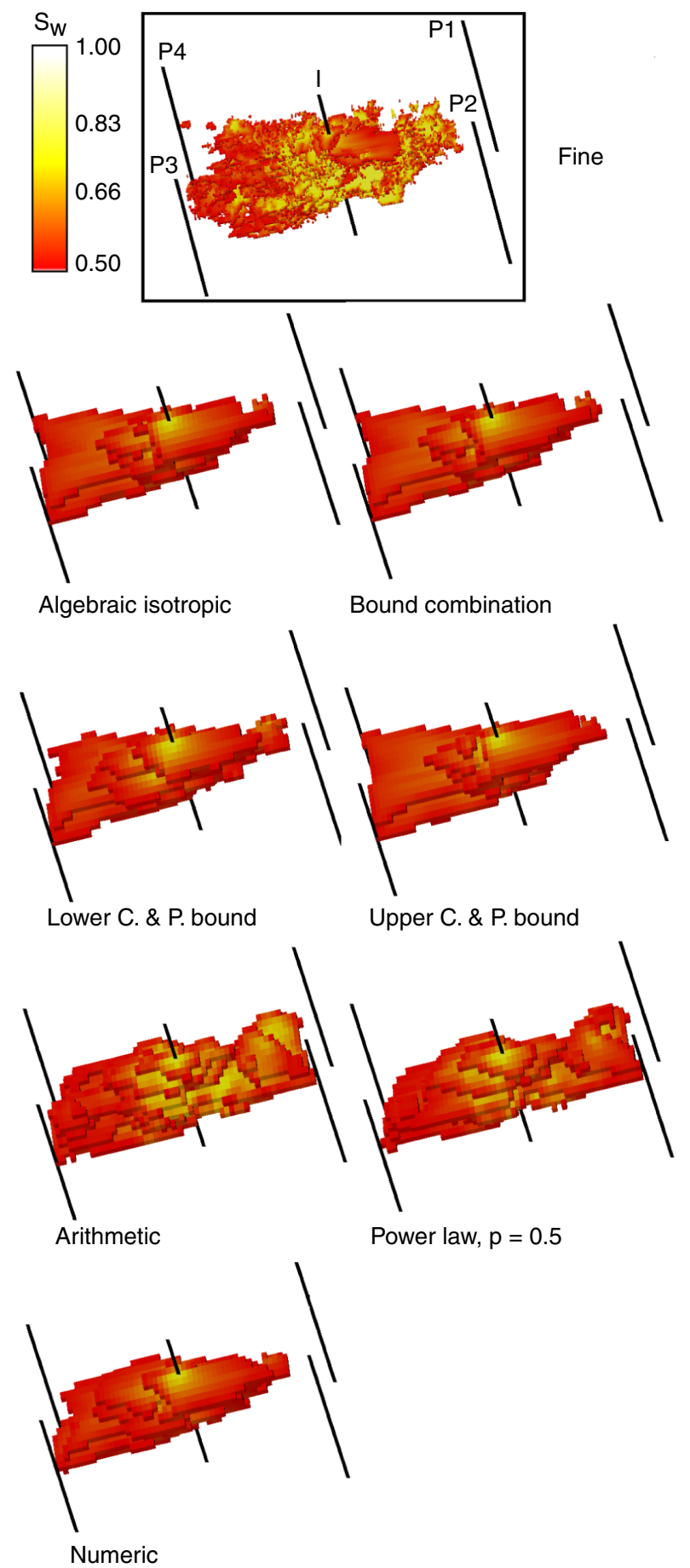

Figure 12

Comparison of water saturation for reservoir models derived from different upscaling methods after 530 days of injection.

We focus on saturation plots to analyze the impact of upscaling. Figure 12 shows water saturation after 530 days of injection.
The upscaling process smooths the displacement front, but its general form is more or less preserved. As expected, the bound combination method and the algebraic isotropic method give the same results in terms of water saturation. As mentioned earlier, anomalous behavior was evidenced for the water-oil ratios simulated for wells P3 and P4. For these two wells, Figure 9 shows that the front is very different from the one obtained on the fine scale. In the case of the oil rate, we observed that the power-law method with an exponent of 0.5 and the arithmetic average lead to similar water displacements. Whatever the upscaling method applied to this test case, the displacement front tends to extend, especially for wells $\mathrm{P} 3$ and $\mathrm{P} 4$. This may be due to coarsening, or more precisely, to numerical diffusion. For wells P1 and P2, the front also extends with the arithmetic mean and the power-law method (with an exponent of 0.5). These two methods are known to be optimistic. Therefore, permeability overestimation adds to numerical diffusion, which accentuates front distortion.

\subsection{How Important is the Choice of the Upscaling Factor?}

Section 3.2 investigated the influence of the choice of the upscaling method on the simulation results. Now, given an upscaling method, we propose to focus on the upgridding step, that is, on the analysis of the upscaling factor impact. The application case considered hereafter is the bottom part of the SPE10 case. This unit includes very thin and long channels. As such, the flow simulation results are expected to be very sensitive to the upscaling factor. The upscaling method used is the bound combination one [25] (Eq. 26).

\subsubsection{The Upscaling Factor and the Upscaling Extent Parameter}

In the first step, we study the relative effect of coarsening in different directions. We envision two different configurations: first along the $X$ direction, and second along the $Y$ direction. The dimensions of the grids used are reported in Tables 4 and 5 along with the upscaling factor values.

The $U E$ Upscaling Extent parameter is computed for the two coarsening configurations of interest. Both global (Eq. 3) and directional (Eq. 5) parameters are represented in Figure 10. The $U E$ decreasing at small upscaling factors is stronger for the upscaling along direction $Y$ than for the upscaling along direction $X$. According to [10], the information loss is proportional to the $U E$. Therefore, the $U E$ curves depicted in Figure 13 can be used to evaluate the information loss. This idea drives our understanding of the results presented in the following subsections. 


\section{TABLE 4}

Number of grid blocks along axis $X\left(N_{x}\right)$ versus the corresponding upscaling factor. Upscaling is applied along the $X$ direction only

$$
\left(N_{y}=220, N_{z}=50\right)
$$

\begin{tabular}{c|c}
\hline$N_{\mathrm{x}}$ & Upscaling factor \\
\hline 30 & 0.5 \\
\hline 20 & 0.333 \\
\hline 15 & 0.25 \\
\hline 12 & 0.2 \\
\hline 10 & 0.166 \\
\hline 6 & 0.1 \\
\hline 5 & 0.0833 \\
\hline 3 & 0.05 \\
\hline 2 & 0.033 \\
\hline
\end{tabular}

TABLE 5

Number of grid blocks along axis $Y\left(N_{y}\right)$ versus the corresponding upscaling factor. Upscaling is applied along the $Y$ direction only $\left(N_{x}=60, N_{z}=50\right)$

\begin{tabular}{c|c}
\hline$N_{\mathrm{y}}$ & Upscaling factor \\
\hline 110 & 0.5 \\
\hline 55 & 0.25 \\
\hline 44 & 0.2 \\
\hline 22 & 0.1 \\
\hline 11 & 0.05 \\
\hline 10 & 0.04545 \\
\hline 5 & 0.022727 \\
\hline 3 & 0.01818 \\
\hline 2 & 0.00909 \\
\hline
\end{tabular}

In the Figure 13, we remark that for the coarsening along direction $X$, the $U E$ and $U E_{x}$ variations are more linear like the $U E$ and $U E_{y}$ variations. Qi and Hesketh [10] stated that the information loss is proportional to the $U E$ criterion. Therefore, these curves can also help explain the next results.

\subsubsection{The Data Range and Variance Indicators}

Before going further, we detail the key features of the selected upscaling method when applied to this special case. As explained above, the bound combination

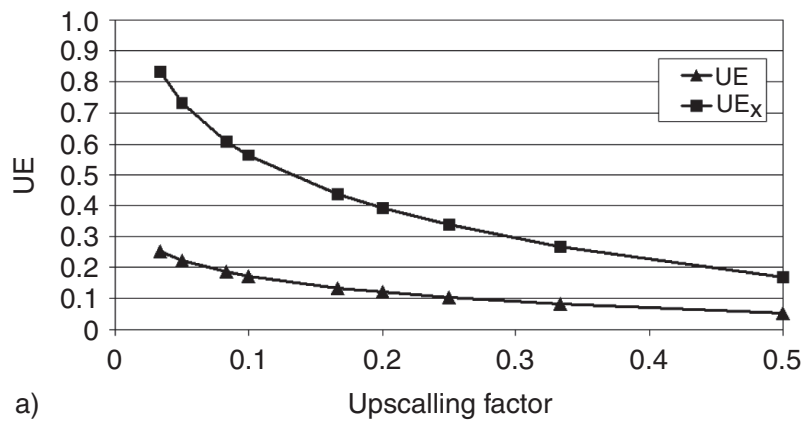

a)

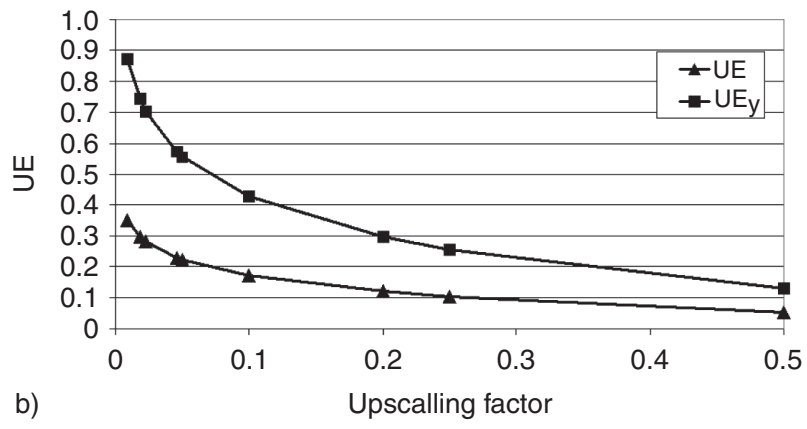

Figure 13

The Upscaling Extent (UE) parameter versus the upscaling factor for the two coarsening directions considered (coarsening along a) axis $X$ only, b) axis $Y$ only). The global $U E$ parameter is plotted together with the directional $U E\left(U E_{x}\right.$ and $U E_{y}$ ).

method is a generalization of the algebraic isotropic method. It depends on the Cardwell and Parsons bounds. The effective permeability, when computed along direction $X$, is given by Equation (26). In the case studied, we upscale the model either along $X$ or $Y$. Then, with the upscaling along $X$, a coarse cell encompasses a single cell along directions $Y$ and $Z$ and several along direction $X$ (Fig. 14). So, if only upscaling in one direction is carried out then it is trivial that the harmonic average might be chosen along the coarse cell and arithmetic average for flow transverse to the cell. In this case, the bound combination method boils down to this configuration.

In fact, based upon this configuration, expressions $(13,14,27)$ and $(28)$ can be simplified, resulting in:

$$
\begin{aligned}
& K_{c, x}^{\min }=K_{c, x}^{\max }=K_{c, x}^{3}=K_{c, x}^{4}=\mu_{h}^{x}\left(K_{f, x}\right) \\
& K_{c, y}^{\min }=K_{c, y}^{\max }=K_{c, y}^{3}=K_{c, y}^{4}=\mu_{a}^{x}\left(K_{f, y}\right) \\
& K_{c, z}^{\min }=K_{c, z}^{\max }=K_{c, z}^{3}=K_{c, z}^{4}=\mu_{a}^{x}\left(K_{f, z}\right)
\end{aligned}
$$




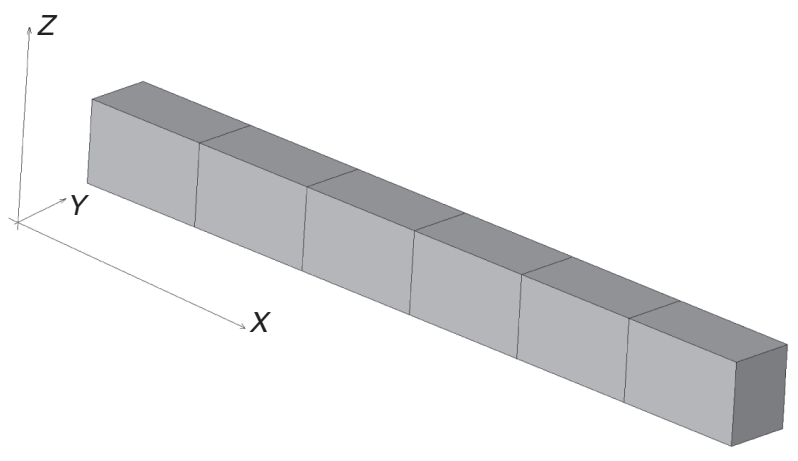

Figure 14

Fine cells included in a coarse cell (upscaling along direction $X$ only; upscaling factor of 0.166 ).

In this case, the bound combination method yields:

$$
\begin{aligned}
& K_{x}^{\text {BoundComb. }}=\mu_{h}^{x}\left(K_{f, x}\right) \\
& K_{y}^{\text {BoundComb. }}=\mu_{a}^{x}\left(K_{f, y}\right) \\
& K_{z}^{\text {BoundComb. }}=\mu_{a}^{x}\left(K_{f, z}\right)
\end{aligned}
$$

As a conclusion, when aggregating cells along direction $X$ only, the bound combination method boils down to the harmonic mean for $K x$ and the arithmetic mean for $K y$ and $K z$. The same result is obtained when considering the algebraic isotropic method instead of the bound combination method. This is a known result in upscaling ([42] p. 30).

Figure 15 shows that the indicator values obtained for $K y$ and $K z$ are pretty close for the upscaling along $X$. Similarly, the results for $K x$ and $K y$ are very close for the upscaling along $Y$. These similarities and the simplification of the bound combination method arise because there is only one direction of upscaling. The variations in the data range indicators provide useful insights about the maximal value that can be used for the upscaling factor. When along $X$, the upscaling process is erroneous for upscaling factors less than 0.25 . Even with an upscaling factor of 0.166 , the smoothing effect due to upscaling can be considered as too strong. When along direction $Y$, the upscaling process is characterized by a critical upscaling factor of 0.1 . The overall variations in the variance indicator being linear, it is difficult to draw conclusion. The only remarkable point is that the values obtained with the harmonic mean are higher than with the arithmetic mean. This is also true for the data range indicator.
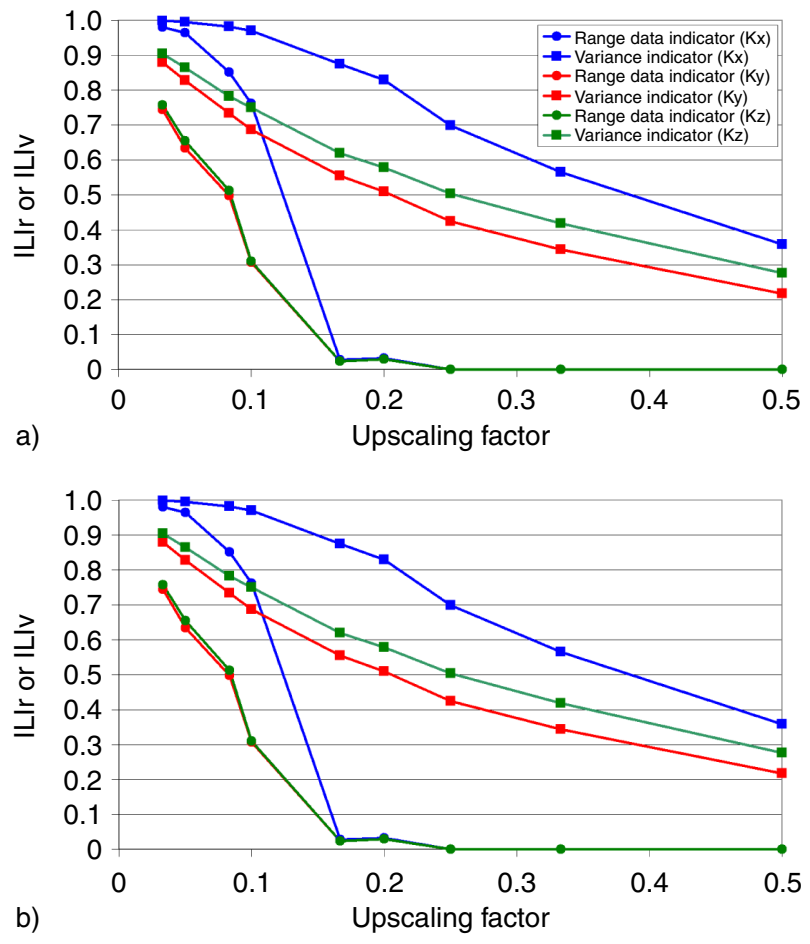

Figure 15

Data range (ILIr) and variance (ILIV) indicators obtained when performing upscaling along a) $X$ and b) $Y$.

\subsubsection{Effects of Coarsening on the QQplot}

Whatever the coarsening direction, Figures 16 and 17 show that the smaller the upscaling factor, the more the QQplot departs from the bisector. In other words, the smaller the upscaling factor, the less accurate the upscaling. In addition, these figures point out that the upscaling process is wrong for the intermediate permeability values in between $1 \mathrm{mD}$ and $10 \mathrm{mD}$, while the extreme values are properly captured. Moreover, as explained previously in the paper the bound combination method boils down to the harmonic mean in the direction of upscaling, and to the arithmetic mean in the other directions. And the harmonic mean will be pessimistic in the direction of upscaling (QQplot below the bisector) and the arithmetic average will be optimistic (QQplot above the bisector). The results obtained for vertical permeabilities are more intricate, especially for high upscaling factors. Two bumps are observed: one when fine permeabilities are in the range $\left[10^{-4} \mathrm{mD} ; 10^{-2} \mathrm{mD}\right]$ and the other one when they are in the range $\left[10^{-1} \mathrm{mD}, 10 \mathrm{mD}\right]$. However, these two bumps merge when the upscaling factor decreases. 


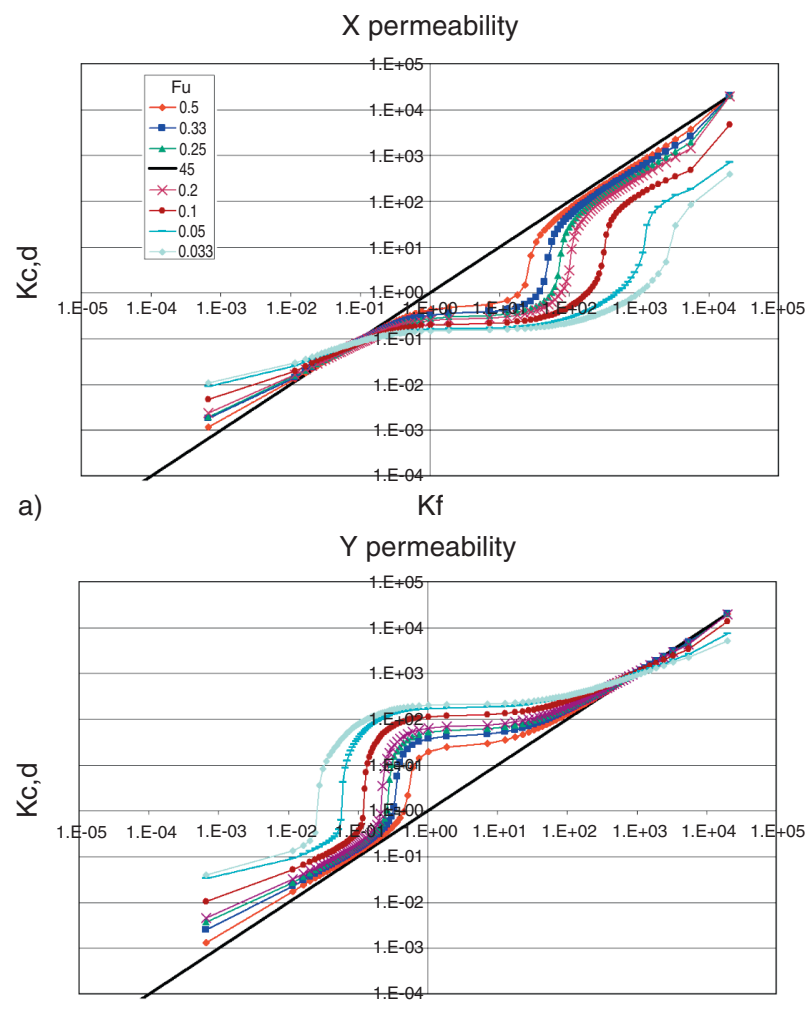

b)

$\mathrm{Kf}$

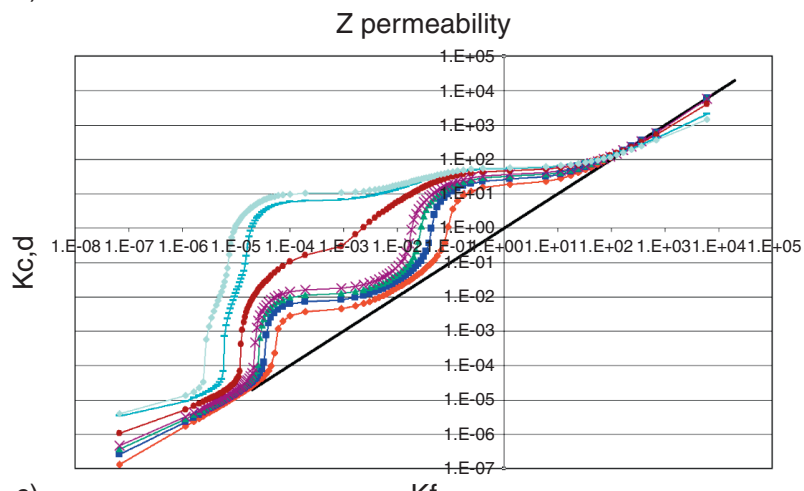

c)

$\mathrm{Kf}$
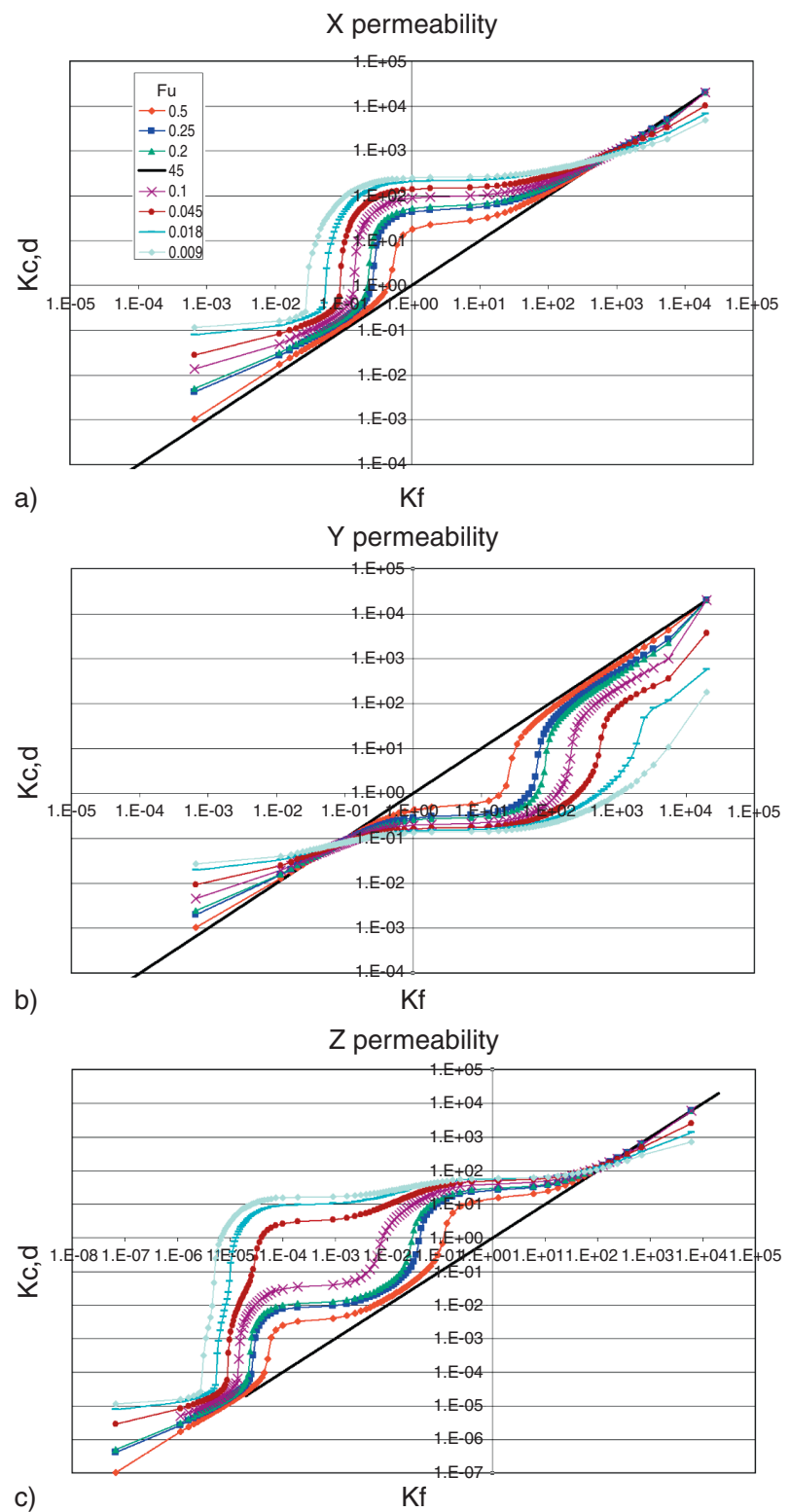

Figure 17

QQplots for a) horizontal $X$, b) horizontal $Y$ and c) vertical $Z$ permeabilities when performing upscaling along $Y$.

This phenomenon related to systems close to their percolation threshold is detailed in [16].

\subsubsection{Convergence of the Quality Indicators and the Simulation Results}

This section presents the production responses simulated for the various reservoir models obtained, considering the two upscaling configurations described above: coarsening along $X$ and coarsening along $Y$.

\section{Coarsening along $X$}

Let us first focus on the responses simulated for the injector, which is located in the middle of the field. Figure 18 depicts the bottom-hole pressure and the water injection rate at surface conditions for the different upscaling factors. As expected, the injection rate is almost constant: the simulator has difficulties maintaining a constant injection rate, but the difference is negligible (a maximal difference of $10 \mathrm{~m}^{3} /$ day for a constant rate of $795 \mathrm{~m}^{3} /$ day). The difference in bottom-hole pressure 

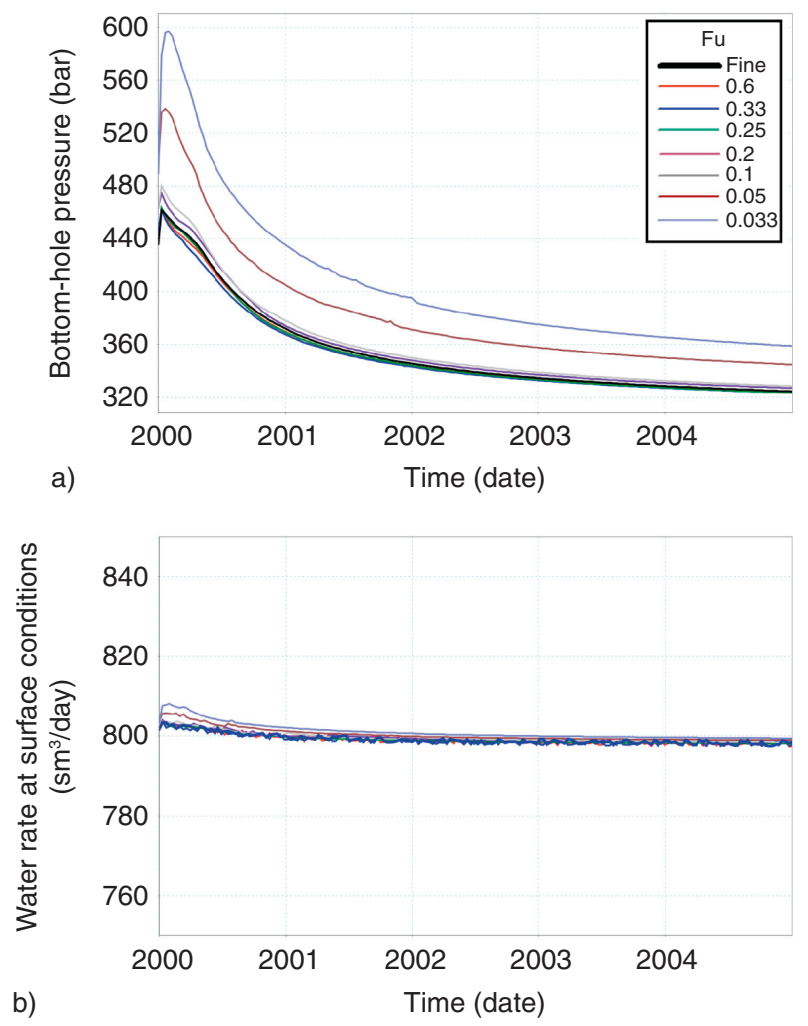

Figure 18

a) Bottom-hole pressures and b) water injection rates at surface conditions at the injector for various upscaling factors - upscaling is performed along direction $X$ (black $=$ fine grid results).

tells us about the average permeability between wells and about the upscaled productivity index. The upscaling modifies this average permeability and the productivity index: we also see a difference in BHP. Small $F u$ upscaling factors lead to very inaccurate results. When $F u<0.1$ (that is $U E>0.17$ or $U E_{x}>0.56$ ), bottom-hole pressures and water injection rates are both overestimated. When $F u>0.16$ (that is $U E<0.134$ or $\left.U E_{x}<0.438\right)$, the fine and coarse simulated production responses are pretty close. This is consistent with the results for the ILIr data range indicator shown in Figure 15. When the upscaling factor is more than 0.16 , the $I L I r$ values obtained indicate very bad results. When $F u$ decreases, that is when the $U E$ increases; the responses simulated for the injector deteriorate. For $F u>0.33$ (for $U E<0.082$ or $U E_{x}<0.27$ ), the results for the coarse models are in very good agreement with the responses simulated for the fine model. Moreover, the water injection rates computed are smoother when $F u \leq 0.25$ (Fig. 18a). This may be induced by the homogenization of the permeability field. Thus, for the case studied and considering only the responses at the injector, the upgridding can be regarded as reasonable as long as the upscaling factor $F u$ is more than 0.33. In the case of the producers, we focused on the water-oil ratios (Fig. 22) as these responses are very sensitive to the upscaling factor. The breakthrough is actually a key factor when managing reservoir production. It is therefore crucial to build upscaled reservoir models able to properly reproduce the breakthrough simulated for the fine model. For a small upscaling factor, we remark that the results are very inaccurate. For $F u<0.1$ $\left(U E>0.17\right.$ or $\left.U E_{x}>0.56\right)$, the bottom-hole pressure and the water injection rate are overestimated. For $F u>0.16\left(U E<0.134\right.$ or $\left.U E_{x}<0.438\right)$ the results are quite similar to the fine results. This conclusion can also be drawn from the ILIr result (Fig. 15). For an upscaling factor higher than 0.16 , the ILIr results are very bad. We remark that when $F u$ lowers and so when the UE increases, the results deteriorate at the injector. The results for $F u>0.33$ (for $U E<0.082$ or $U E_{x}<0.27$ ) are in very good agreement with the fine results. Moreover, the water injection rate results are smoother for $F u \leq 0.25$ : this may be due to the homogenization of the permeability field. As a conclusion, the upgridding is reasonable for the injector results when $F u>0.33$. This value gives an idea of the Representative Elementary Volume of this case. This also has to be investigated (Fig. 19) for the responses simulated at the producer. For the four producers, the results are very poor when $F u<0.16\left(U E>0.134\right.$ or $\left.U E_{x}>0.438\right)$. This is the same as for the injector. Second, the results obtained for wells P2, P3 and P4 are in very good agreement with the fine results when $F u>0.33$ (for $U E<0.082$ or $U E x<0.27)$. However, for well $\mathrm{P} 1$, all results are relatively bad whatever the upscaling factor: too much water is produced at this well. The upscaled permeabilities around this well are probably overestimated. Despite these poor results for well $\mathrm{P} 1$, we concluded that the breakthrough was correctly captured provided $F u$ was more than 0.33 .

\section{Coarsening along $Y$}

The same study was performed when performing the upscaling along the $Y$ direction. The bottom-hole pressures and the water rates simulated for the injector are displayed in Figure 20. It is worth noting that the maximal bottom-hole pressure is 689.18 bar (10 $000 \mathrm{psi})$.

Figure 20 shows that the injector reaches the maximal bottom-hole pressure when $F u<0.05$ (UE $>0.224$ or $U E_{y}>0.556$ ). At this point, as soon as the pressure constraint is reached, the well production is based upon a 

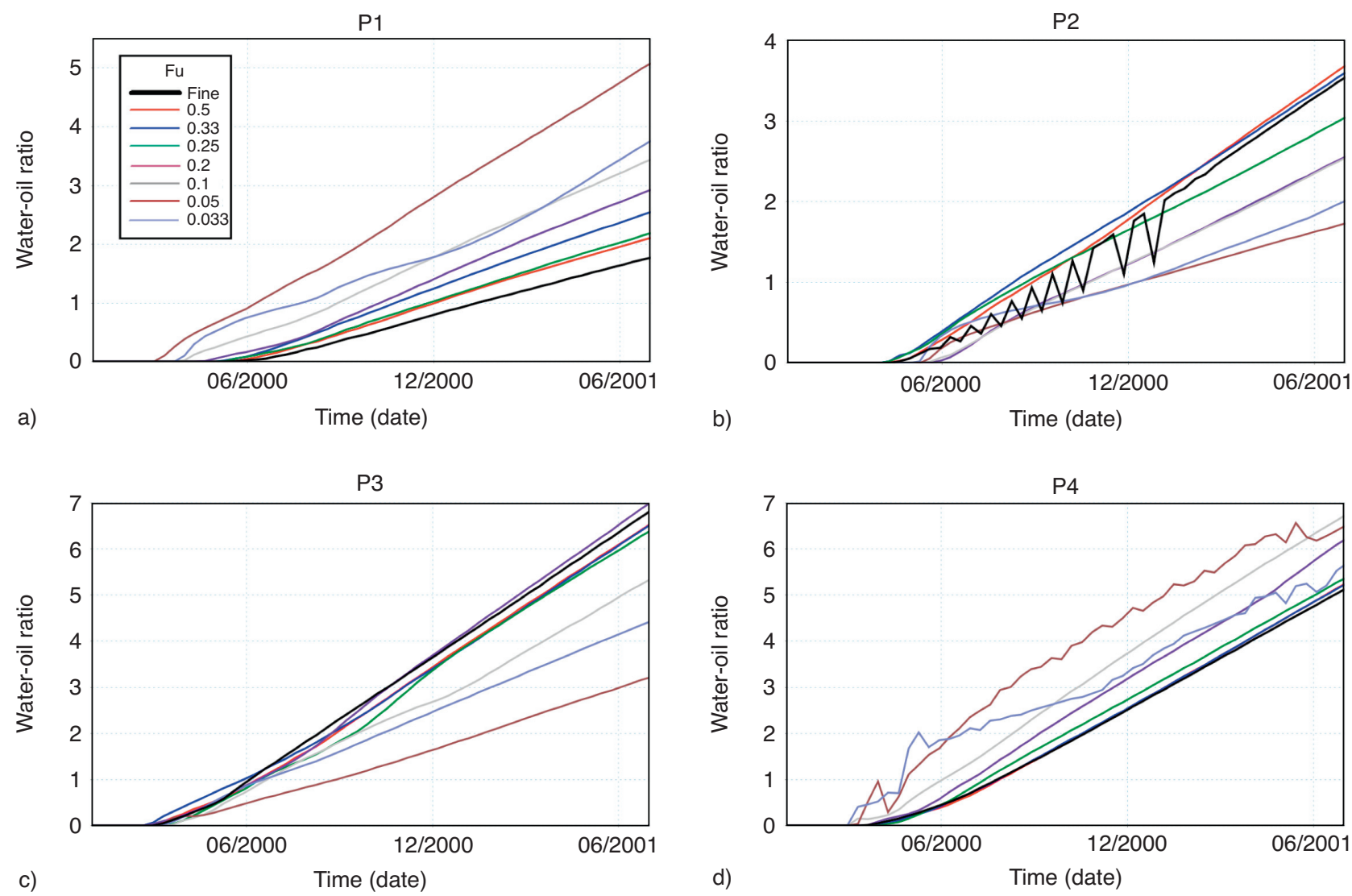

Figure 19

Water-oil ratios at producers a) P1, b) P2, c) P3 and d) P4 for various upscaling factors - upscaling is performed along direction $X$ (black $=$ fine grid results).

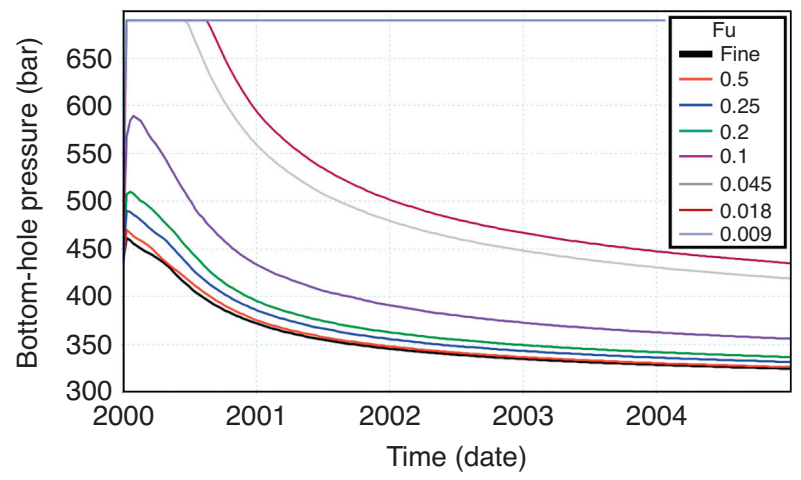

Figure 20

Bottom-hole pressures at the injector for various upscaling factors - upscaling is performed along direction $Y$ (black $=$ fine grid results).

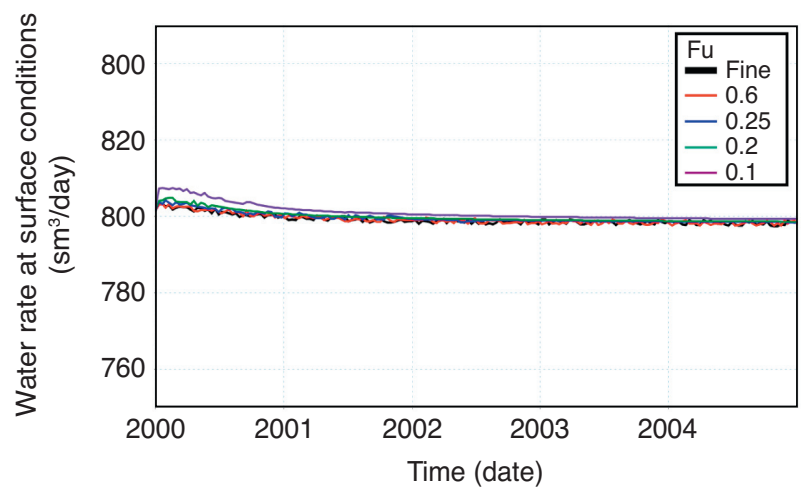

Figure 21

Constant water injection rate for various upscaling factors - upscaling is performed along direction $Y$ (black $=$ fine grid results). 

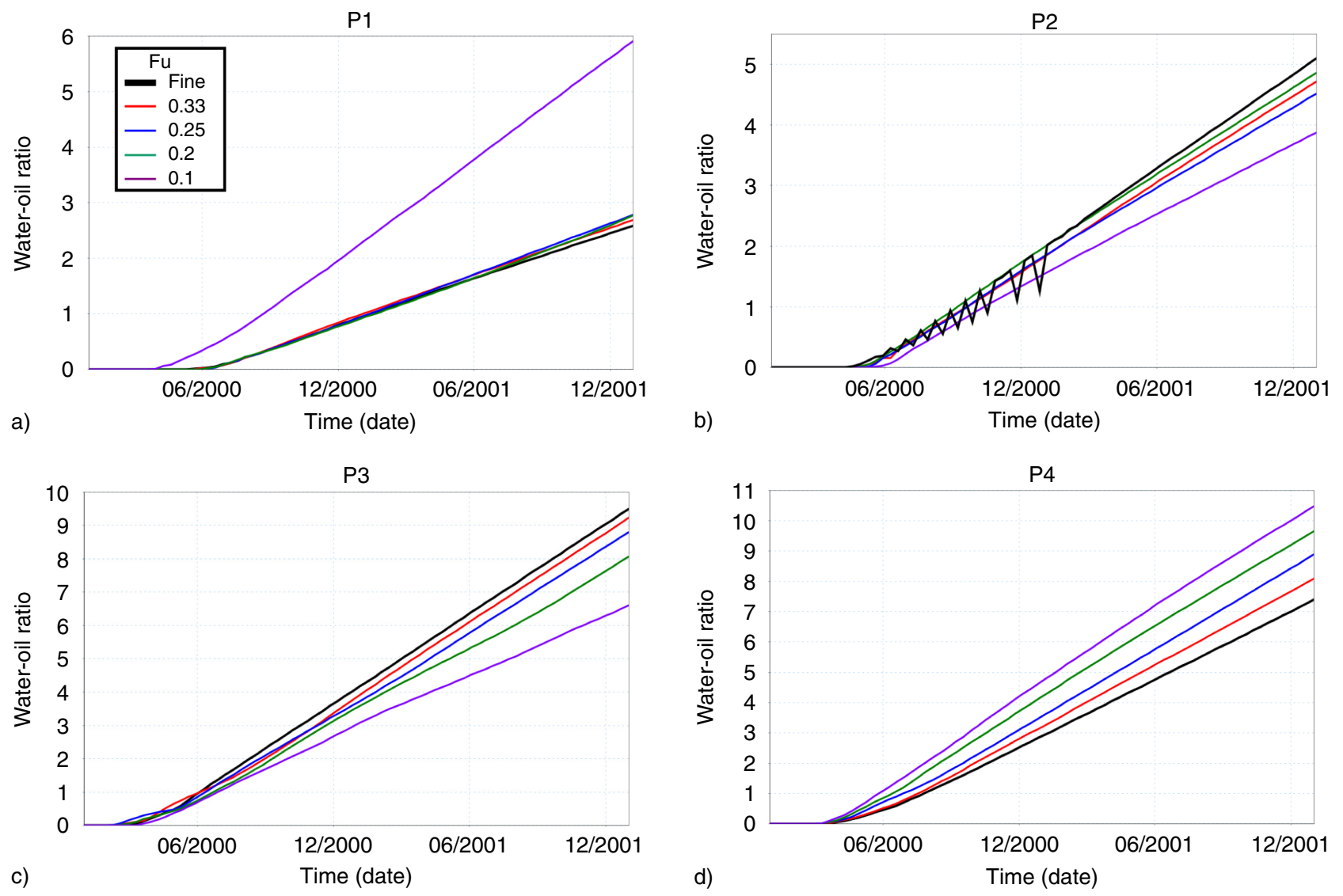

Figure 22

Water-oil ratios for various upscaling factors - upscaling is performed along direction $Y$ (black = fine grid results).

constant pressure and the water injection rate falls. In such conditions, there is no way to make valuable comparisons. A major result is that too strong a coarsening can drastically change the dynamic behavior of the field. This phenomenon could be predicted from the analysis of the data range indicators. Figure 15 shows that ILIr values strongly decrease for upscaling factors within the range $[0.05 ; 0.1]$. When $F u$ is more than 0.01 , the ILIr values are very small. Another insight can also be detected from the analysis of QQplots for vertical permeabilities (Fig. 17). When Fu<0.05, the shape of the QQplots changes and the two small bumps combine to form a single one. Based upon this result, we restrict our attention to the water injection rates simulated for $F u>0.05$.

Figure 21 shows that water injection rates are lightly overestimated when $F u$ is too small. The simulator has difficulties maintaining a constant injection rate (oscillations), but the difference is negligible (a maximal differ- ence of $10 \mathrm{~m}^{3} /$ day for a constant rate of $795 \mathrm{~m}^{3} /$ day). In addition, the upscaling process contributes to homogenizing permeabilities. Thus, the smaller $F u$, the smoother the results. We can also observe that the results are very good when $F u>0.2$. When $F u=0.1$, the rates simulated for the upscaled models are not very accurate, but the main features of well behavior are preserved.

In the case of the producers, we focus on the water-oil ratios. At first glance, the results obtained for $F u=0.1$ are wrong. This is particularly obvious for well P1. For this problematic well, the results turn out to be correct as soon as $F u \geq 0.2$. For wells P3 and P4, the simulated water-oil rates clearly depend on $F u$ : the smaller the $F u$ (the higher the UE, ILIr and ILIem), the worse the results. Depending on the criterion considered, the water-oil ratio is underestimated for well P3 while it is overestimated for well $\mathrm{P} 4$. As a conclusion, the upgridding is reasonable when $F u \geq 0.2$ : to obtain correct results we must choose an upscaling factor greater than 0.2 . 


\section{CONCLUSION}

In this paper, we studied and detailed different criteria, which make it possible to evaluate the validity of upscaling methods and to quantify the information loss resulting from their application. We studied two test cases, the first one to see whether indicators or criteria help to identify the best upscaling method to use, and the second one to identify the most relevant upscaling factor. We showed that the QQplot and the variance indicator are valuable preprocessing tools to investigate the impact of uspcaling before performing any fluid flow simulation. Then, estimating the Cardwell and Parsons bounds helps identify the cells or directions for which upscaling may lead to erroneous results if not carefully and properly done. The analysis of Cardwell and Parsons bounds combined together with the search for the zero data range and variance indicators provide useful insights into the validity of the upscaled results obtained. In addition, we observed that for the first test case the bound combination, algebraic and numerical local methods give the most satisfactory results. Combining these methods to upscale the absolute permeabilities with a method to upscale well productivity indices [43] would contribute to improving the results further. The second test case pointed out that these different criteria are also very useful for selecting an appropriate upscaling factor. In this case, an upscaling factor greater than 0.2 gives correct results in terms of the oil rate or water-oil ratio. This study treats one example of heterogeneous medium: the well-known SPE10 test case. A perspective of this work could be to test how these different upscaling indicators suit different types of heterogeneity.

\section{ACKNOWLEDGMENTS}

The author would like to thank Dr. Mickaele Le Ravalec-Dupin for her valuable help and advice in writing this paper.

\section{REFERENCES}

1 Durlofsky L.J. (2003) Upscaling of geocellular models for reservoir flow simulation: a review of recent progress, Proceedings of the 7th International Forum on Reservoir Simulation, Buhl/Baden-Baden, Germany, 23-27 June.

2 Farmer C.L. (2002) Upscaling: a review, International Journal for Numerical Methods in Fluids 40, 63-78

3 Renard Ph., de Marsily G. (1997) Calculating equivalent permeability: a review, Advances in Water Resources 20, 5-6, 253-278.
4 Wen X.H., Gómez-Hernández J.J. (1996) Upscaling hydraulic conductivities in heterogeneous media: an overview, Journal of Hydrology 183, ix-xxxii.

5 Sánchez-Vila X., Guadagnini A., Carrera J. (2006) Representative hydraulic conductivities in saturated groundwater flow, Reviews of Geophysics 44, 3.

6 Sablok C., Aziz K. (2005) Upscaling and discretization errors in reservoir simulation, SPE simulation symposium, Houston, Texas, USA, 31 Jan.-2 Feb., SPE 93372.

7 Lodder R., Hieftje G. (1988) Quantile Analysis: a method for characterizing data distributions, Applied Spectroscopie 42, section 8 .

8 Preux C., (2011) Study of evaluation criteria for reservoir upscaling, 73rd EAGE Conference \& Exhibition incorporating SPE EUROPEC 2011, Vienna, Austria, 23-26 May.

9 Qi D. (2010) Upscaling Extent vs. Information Loss in Reservoir Upscaling, Petroleum Science and Technology 28, 12, 1197-1202.

10 Qi D., Hesketh T. (2004) Quantitative Evaluation of Information Loss in Reservoir Upscaling, Petroleum Science and Technology 22, 11, 1625-1640.

11 Qi D., Wong P.M., Liu K. (2001) An Improved Global Upscaling Approach for Reservoir Simulation, Petroleum Science and Technology 19, 7, 8, 779-795.

12 Christie M.A., Blunt M.J. (2001) Tenth SPE Comparative Solution Project: A comparison of Upscaling Techniques. SPE66599, SPE Reservoir Simulation Symposium, Houston, Texas, 11-14 Feb.

13 Gautier Y., Blunt M.J., Christie M. A. (1999) Nested gridding and streamline-based simulation for fast reservoir performance prediction, Computational Geosciences 3, 3-4, 295-320.

14 Salazar M.O., Villa J.R. (2007) Permeability upscaling techniques for reservoir simulation. Latin American \& Caribbean Petroleum Engineering Conference, Buenos Aires, Argentina, 15-18 April, SPE106679.

15 Fleckenstein J.H., Fogg G.E. (2008) Efficient upscaling of hydraulic conductivity in heterogeneous alluvial aquifers, Hydrogeology Journal 16, 7, 1239-1250.

16 Boschan A., Noetinger B., (2012) Scale dependence of effective hydraulic conductivity distributions in 3D heterogeneous media: a numerical study, Transport in Porous Media 94, 1, 101-121.

17 Hosseini S.A., Kelkar M. (2008) Analytical Upgridding method to preserve dynamic flow behavior, SPE Annual Technical Conference and Exhibition, Denver, 21-24 Sept., SPE 116113.

18 King M.J., Burn K.S., Wang P., Muralidharan V., Alvardo F., Ma X., Datta-Gupta A. (2006) Optimal Coarsening of 3D Reservoir Models for Flow Simulation, SPE Reservoir Evaluation \& Engineering 9, 4, 317-334.

19 Deschamps R., Guy N., Preux C., Lerat O. (2011) Impact of Upscaling on 3D Modelling of SAGD in a Meander Belt, SPE Annual Technical Conference and Exhibition 4, 3328-3343, Society of Petroleum Engineers - Denver, Colorado, USA, 30 Oct.-2 Nov., SPE 147035.

20 Cardwell W.T., Parsons R.L. (1944) Average permeabilities of heterogeneous oil sands, Transactions of the American Institute of Mining, Metallurgical and Petroleum Engineers 160, 34-42. 
21 Renard Ph., Le Loc'h G., Ledoux E., de Marsily G., Mackay R. (2000) A fast algorithm for the estimation of the equivalent hydraulic conductivity of heterogeneous porous media, Water Resources Research 36, 12, 3567-3580.

22 Li D., Kumar A. (2001) A New Efficient Averaging Technique for Scaleup of Multimillion-Cell Geologic Models, SPE Reservoir Evaluation \& Engineering 4, 4, August.

23 Journel A.G., Deutsch C.V., Desbarats A.J. (1986) Power averaging for Block effective permeability. 56th California Regional meeting of SPE, Oakland, SPE 15128.

24 de Dreuzy J.-R., de Boiry P., Pichot G., Davy P. (2010) Use of power averaging for quantifying the influence of structure organization on permeability upscaling in on-lattice networks under mean parallel flow, Water Resour. Res. 46 W08519.

25 Guérillot D., Rudkiewicz J.L., Ravenne C., Renard G., Galli A. (1990) An integrated model for computer aided reservoir description: from outcrop study to fluid flow simulations, Revue de l'IFP 45, 1.

26 Duquerroix J-P.L., Lemouzy P., Noetinger B., Romeu R.K. (1993) Influence of the Permeability Anisotropy Ratio on Large-Scale Properties of Heterogeneous Reservoirs, 68th Annual Technical Conference and Exhibition of the Society of Petroleum Engineers, Houston, Texas, 3-6 Oct., SPE 26612.

27 Kruel-Romeu R. (1994) Écoulement en milieu poreux hétérogènes: prise de moyenne de perméabilités en régime permanent et transitoire (fluid flow through heterogeneous media: averaging permeabilities for the steady-state and transient cases), PhD Dissertation, University of Paris VI.

28 Christie M.A. (1996) Upscaling for reservoir simulation, Journal of Petroleum Technology 48, 11, 1004-1010.

29 Noetinger B., Zargar G. (2004) Multiscale Description and Upscaling of Fluid Flow in Subsurface Reservoirs, Oil \& Gas Science and Technology, Revue de l'IFP 59, 2, 119-139.

30 Pickup G.E., Hern C.Y. (2002) The Development of Appropriate Upscaling Procedures, Transport in Porous Media 46, 119-138.

31 Pickup G.E., Ringrose P.S., Jensen J.L., Sorbie K.S. (1994) Permeability tensors for sedimentary structures, Mathematical Geology 26, 2, 227-250.

32 Jikov V.V., Kozlov S.M., Oleinik O.A. (1994) Homogenization of Differential Operator and Integral Functionals, Springer Verlag
33 Web site: www.spe.org/csp/datasets/set02.htm

34 Brooks R.H., Corey A.T. (1964) Hydraulic properties of porous media, Hydrological Papers (3), Colorado State University.

35 Gratien J.M., Guignon T., Magras J.F., Quandalle P., Ricois O. (2007) Scalability and Load Balancing Problems in Parallel Reservoir Simulation, SPE Reservoir Simulation Symposium, Houston, Texas, 26-28 Feb., SPE 106023.

36 Web site: http://www.beicip.com/index.php/eng/software/ openflowplatform

37 Preux C., McKee F. (2011) Study and Approximation of IMPES Stability: the CFL Criteria, Proceeding of the 6th International Symposium on Finite Volumes for Complex Applications, 6-10 Juin, Pragues, Czech Republic, Fort J., Fürst J., Halama J., Herbin R., Hubert F. (eds), Springer, Vol. 1, pp. 713-721.

38 Wiener O. (1912) Abhandlungen des mathematisch, Physischen Klasse des Königlichen Sächsischen Gesellschaft des Wissenschaften 32, 509.

39 Dagan G. (1979) Models of groundwater flow in statistically homogeneous porous formations, Water Resources Research 15, 1, 47-63.

40 Matheron G. (1967) Éléments pour une Théorie des Milieux Poreux, Masson, Paris.

41 Le Loc'h G. (1987) Étude de la composition des perméabilités par des méthodes variationnelles (Study of the composition of permeabilities using variational methods), $P h D$ Dissertation, École Nationale Supérieure des Mines de Paris.

42 Hunt G.H. (2005) Percolation theory for flow in porous media, Lecture Notes in Physics 674, 67-87, Springer, Berlin Heidelberg.

43 Preux C., Ding D., Duval T. (2010) A new method of nearwell upscaling for reservoir simulations based on optimization, Third International Conference on Porous Media and its applications in Science, Engineering and Industry, Montecatini, Italia, 20-25 June.

Manuscript accepted in April 2014 Published online in July 2014 